\title{
Rapidity Dependent Transverse Momentum Spectra of Heavy Quarkonia Produced in Small Collision Systems at the LHC
}

\author{
Li-Na Gao, ${ }^{1}$ Fu-Hu Liu $\mathbb{D}^{\mathbb{D}},{ }^{2}$ and Bao-Chun Li ${ }^{2}{ }^{2}$ \\ ${ }^{1}$ Department of Physics, Taiyuan Normal University, Jinzhong, Shanxi 030619, China \\ ${ }^{2}$ Institute of Theoretical Physics and State Key Laboratory of Quantum Optics and Quantum Optics Devices, \\ Shanxi University, Taiyuan, Shanxi 030006, China \\ Correspondence should be addressed to Fu-Hu Liu; fuhuliu@163.com
}

Received 14 March 2019; Accepted 5 May 2019; Published 22 May 2019

Academic Editor: Carlos Pajares

Copyright (C) $2019 \mathrm{Li}-\mathrm{Na}$ Gao et al. This is an open access article distributed under the Creative Commons Attribution License, which permits unrestricted use, distribution, and reproduction in any medium, provided the original work is properly cited. The publication of this article was funded by SCOAP $^{3}$.

The rapidity dependent transverse momentum spectra of heavy quarkonia ( $J / \psi$ and $\Upsilon$ mesons) produced in small collision systems such as proton-proton $(p p)$ and proton-lead $(p-\mathrm{Pb})$ collisions at center-of-mass energy (per nucleon pair) $\sqrt{s}\left(\sqrt{s_{\mathrm{NN}}}\right)=5-13 \mathrm{TeV}$ are described by a two-component statistical model which is based on the Tsallis statistics and inverse power-law. The experimental data measured by the LHCb Collaboration at the Large Hadron Collider (LHC) are well fitted by the model results. The related parameters are obtained and the dependence of parameters on rapidity is analyzed.

\section{Introduction}

The study of high energy proton-proton, proton-nucleus, and nucleus-nucleus collisions [1-5] can provide a unique opportunity for ones to understand the strong interaction theory and nuclear reaction mechanism [6-10] and analyze the evolution processes of interacting system and quarkgluon plasma (QGP). At the same time, by this study, one can examine the standard model and other phenomenological models or statistical methods [11-14] and search for new physics beyond the standard model. This study also provides new information for people to understand the origin of the universe. As the basic element in nuclear collisions, proton-proton collisions are worth studying. Meanwhile, as a transition from proton-proton collisions to nucleus-nucleus collisions, proton-nucleus collisions are also worth studying.

With the development of modern experimental and detecting technology, the collision energy has been continuously improved. Meanwhile, more and more information about collision process can be accurately measured in experiments [15-19]. Because the collision time of interacting system is very short, one can only analyze the characteristics of final particles produced in the collisions to obtain the mechanisms of nuclear reactions and the properties of formed matter such as QGP.

Generally, the information of nuclear reactions in experiments can be obtained by measuring the transverse momentum spectrum and correlation, pseudorapidity or rapidity spectrum and correlation, anisotropic flow distribution and correlation, multiplicity distribution and correlation, nuclear modified factor, and so forth [15-19]. The transverse momentum spectrum is one of the most general objects in the study. It is measured by experiments and provides information about temperature and excitation degree of interacting system at the stage of kinetic freeze-out. Therefore, the study of transverse momentum spectrum of final particles is greatly significative in analyzing the mechanisms of nuclear reactions and the properties of QGP.

Many theoretical models and formulas have been applied for the descriptions of transverse momentum spectra. These models and formulas include, but are not limited to, the Boltzmann-Gibbs statistics [1-3], Lévy distribution [4, 5], Erlang distribution [6], Tsallis statistics [7-14], and so on. In this paper, we use a two-component statistical model to describe the experimental transverse momentum spectra of heavy quarkonia $(J / \psi$ and $\Upsilon$ mesons) produced in small 


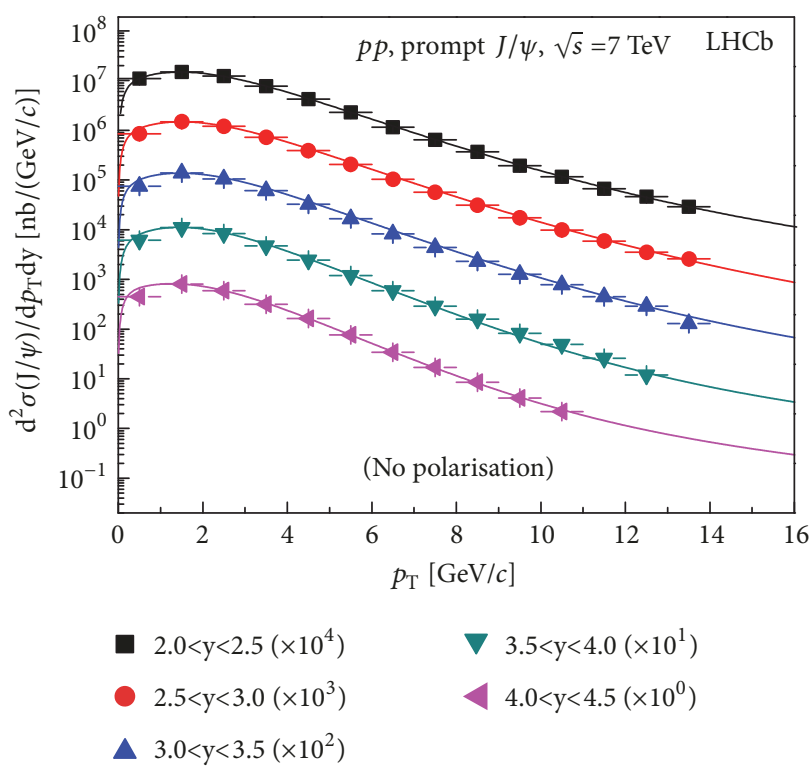

(a)

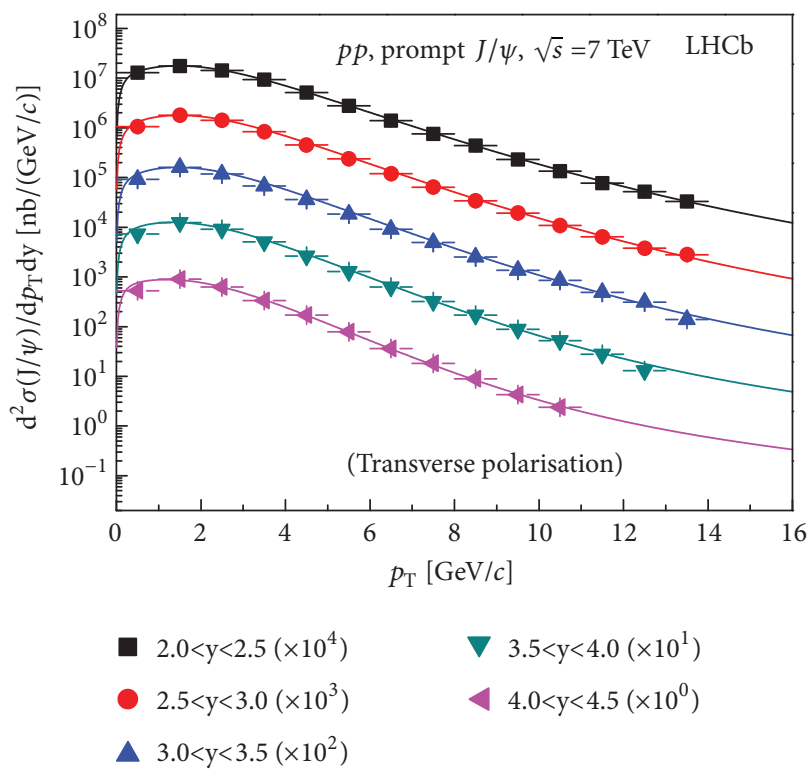

(c)

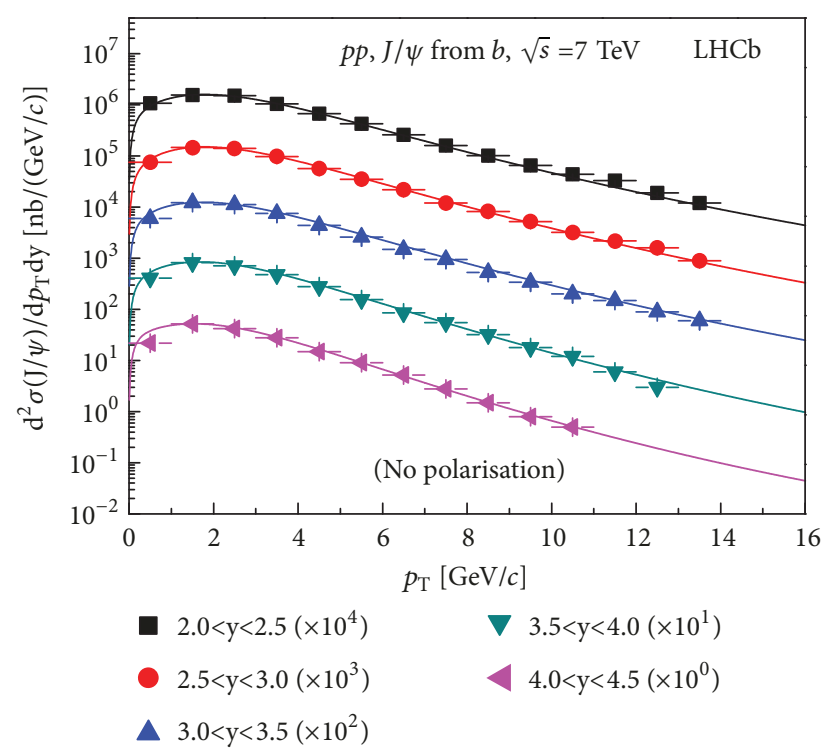

(b)

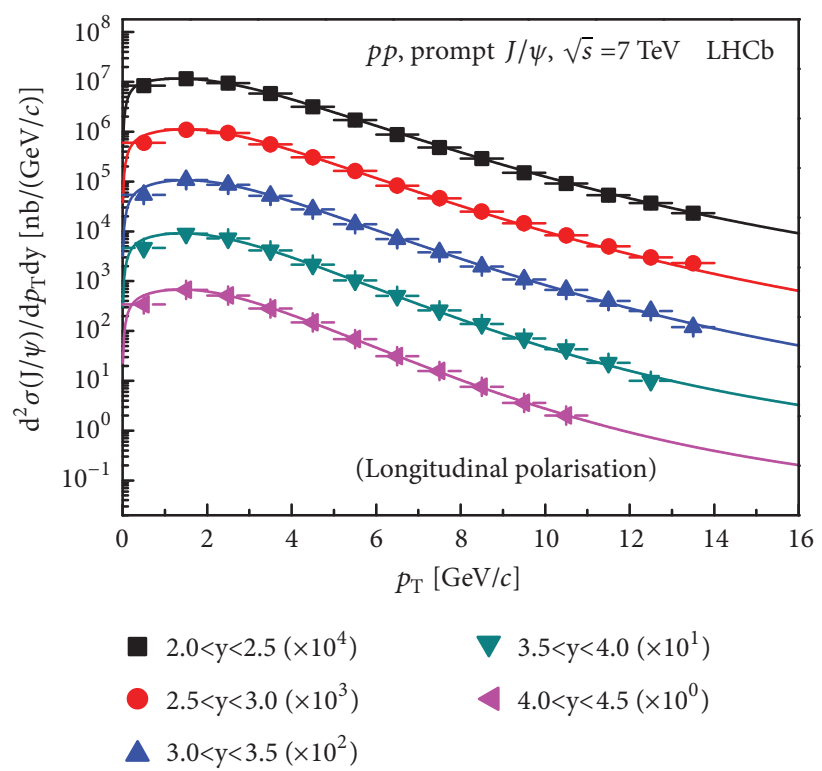

(d)

FiguRE 1: Transverse momentum spectra of (a) prompt $J / \psi$ with no polarisation, (b) $J / \psi$ from $b$ with no polarisation, (c) prompt $J / \psi$ with full transverse polarisation, and (d) prompt $J / \psi$ with full longitudinal polarisation in $p p$ collision at $\sqrt{s}=7 \mathrm{TeV}$. The symbols with the error bars represent the experimental data with the quadratic sums of the statistical and systematic uncertainties measured by the LHCb Collaboration [15] in different rapidity ranges and scaled by different amounts marked in the panels. The curves are our fitted results.

collision systems such as proton-proton $(p p)$ and protonlead $(p-\mathrm{Pb})$ collisions. The data quoted by us are measured by the LHCb Collaboration [15-18] at the Large Hadron Collider (LHC), though other data are available [19]. The twocomponent statistical model is based on the Tsallis statistics and inverse power-law.

In the following sections, we describe the formulism of the two-component statistical model in Section 2. The results and discussion are given in Section 3. Finally, the conclusions of the present work are given in Section 4.

\section{The Formulism}

Within the framework of the multisource thermal model [2022], the emission sources of final particles produced in high energy collisions can be divided into several groups due to different interacting mechanisms, impact parameter ranges (centrality classes), or event samples. A typical classification is soft excitation and hard scattering processes [23-26], and even including very-soft excitation and very-hard scattering processes. Generally, one can use different models and 


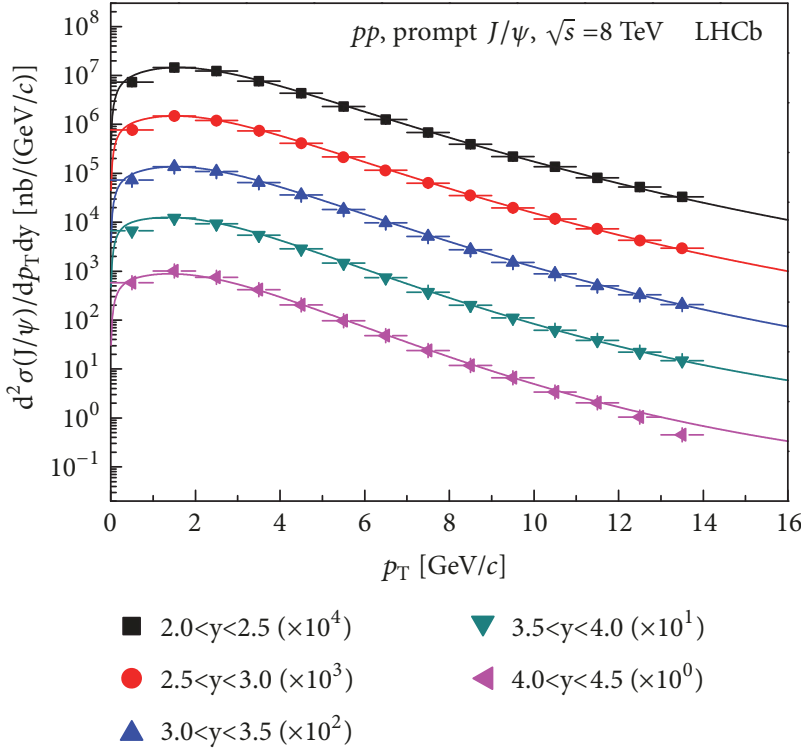

(a)

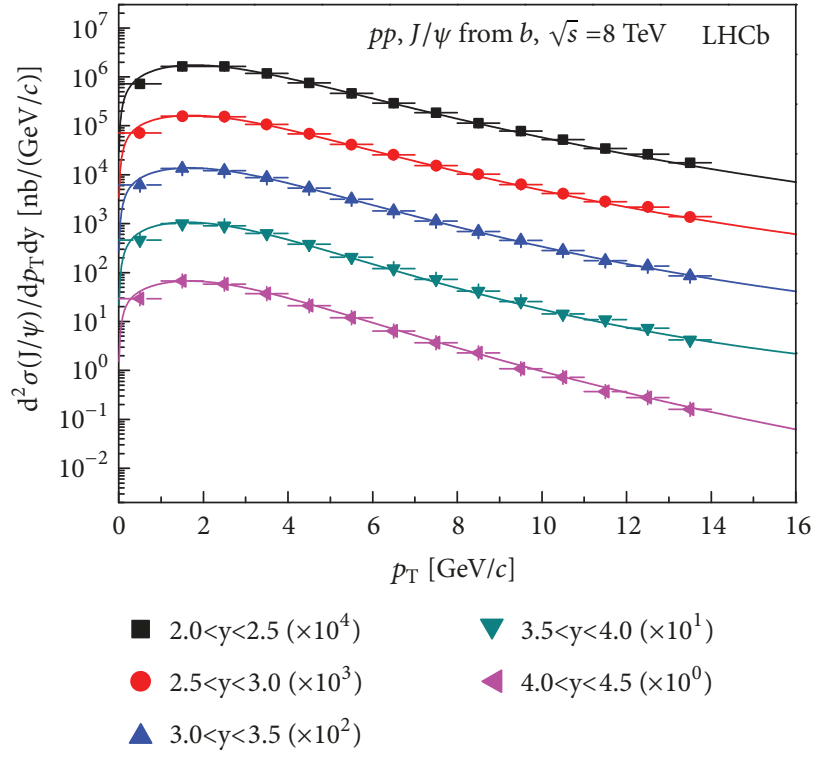

(b)

Figure 2: Transverse momentum spectra of (a) prompt $J / \psi$ and (b) $J / \psi$ from $b$ in $p p$ collisions at $\sqrt{s}=8 \mathrm{TeV}$. The symbols with the error bars represent the experimental data with the quadratic sums of the statistical and systematic uncertainties measured by the LHCb Collaboration [16]. The curves are our fitted results.

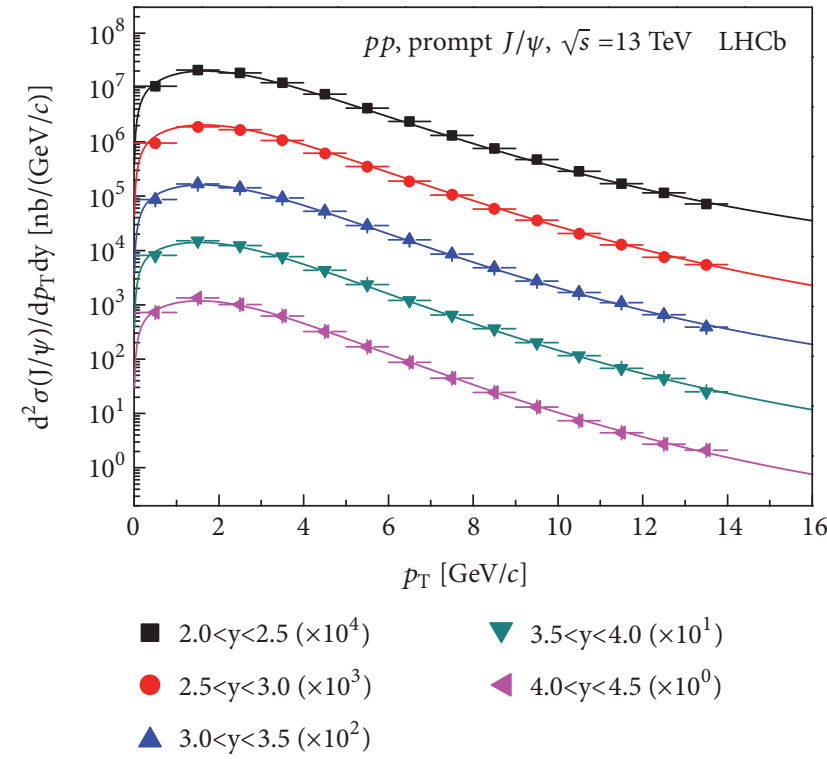

(a)

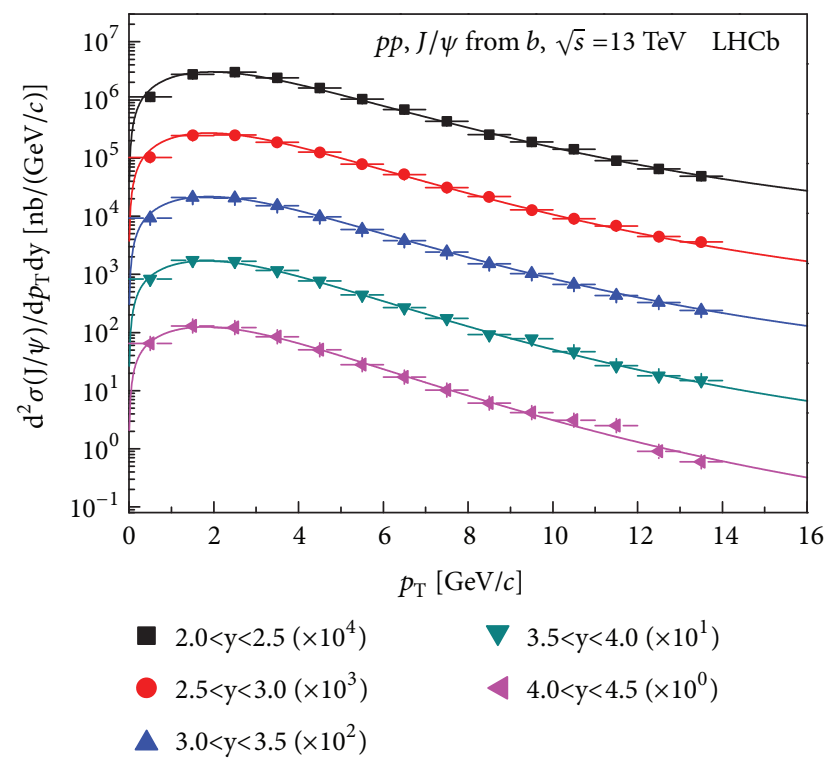

(b)

FIgURE 3: The same as Figure 2, but showing the results of $J / \psi$ mesons produced in $p p$ collisions at $\sqrt{s}=13 \mathrm{TeV}$. The data with the statistical uncertainties are quoted from [18].

formulas to describe different processes. In some cases, one can use the same model and formula to describe different processes. In other cases, one can use different models and formulas to describe the same process.

The Tsallis statistics has been widely applied for high energy collisions [27-31]. It describes different particle spectra in different processes, but not the heavy quarkonium spectra in very-hard process in some cases. For the soft and very-soft processes, the Boltzmann-Gibbs statistics [1-3] also play a main role in the description. For the hard and veryhard processes, an inverse power-law [32-35] play the main role in the description. For the transverse momentum $\left(p_{T}\right)$ spectra of heavy quarkonia $(J / \psi$ and $\Upsilon$ mesons) produced in collisions at the LHC, we need a superposition of the Tsallis statistics and the inverse power-law, which is a twocomponent statistical model. 


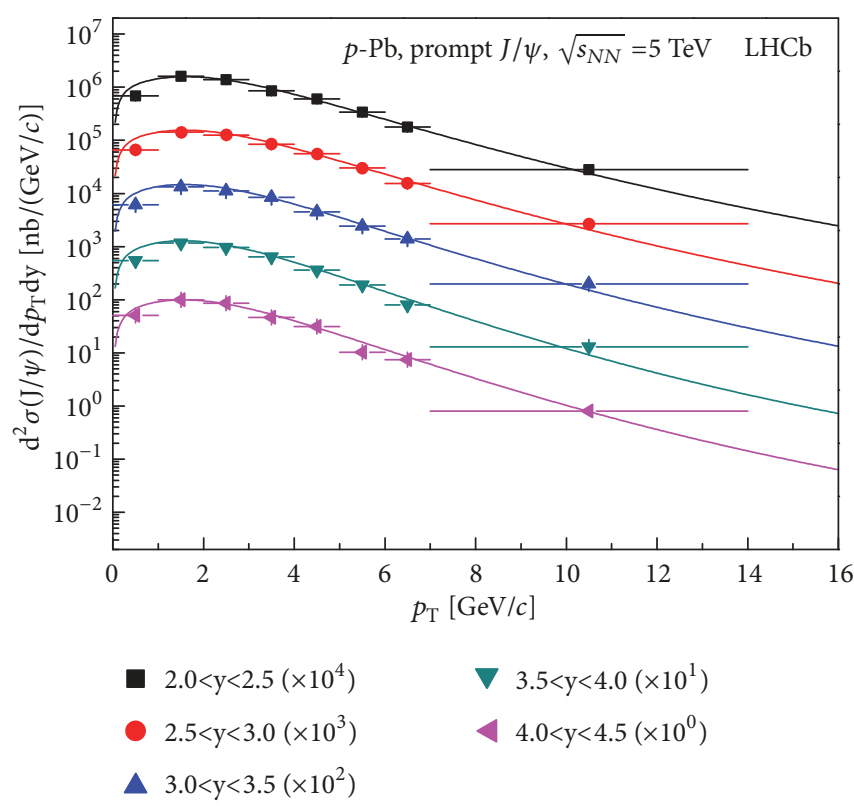

(a)

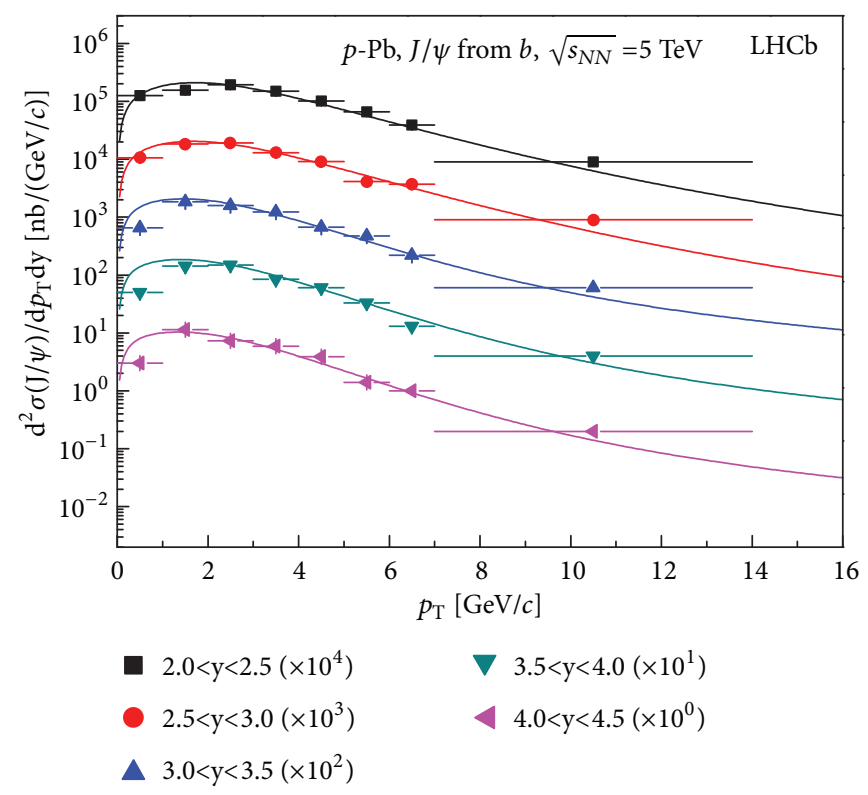

(b)

FIgURE 4: The same as Figure 2, but showing the results of $J / \psi$ mesons in $p$ - $\mathrm{Pb}$ collisions at $\sqrt{s_{\mathrm{NN}}}=5 \mathrm{TeV}$. The data with the quadratic sums of the statistical and systematic uncertainties are quoted from [17].

In the Tsallis statistics [27-30], the invariant momentum (p) distribution is

$$
\begin{aligned}
& E \frac{d^{3} N}{d p^{3}}=\frac{g V}{(2 \pi)^{3}} \\
& \quad m_{T} \cosh y\left[1+\frac{q-1}{T}\left(m_{T} \cosh y-\mu\right)\right]^{-q /(q-1)},
\end{aligned}
$$

where $E$ is the energy, $N$ is the particle number, $g$ is the degeneracy factor, $V$ is the volume, $m_{T}=\sqrt{p_{T}^{2}+m_{0}^{2}}$ is the transverse mass, $m_{0}$ is the rest mass, $T$ is the temperature parameter, $q$ is the entropy index, and $\mu$ is the chemical potential. The normalized $p_{T}$ distribution can be given by

$$
\begin{aligned}
\frac{1}{N} & \frac{d N}{d p_{T}}=\frac{g V}{(2 \pi)^{2}} p_{T} m_{T} \int_{y_{\min }}^{y_{\max }} \cosh y[1 \\
& \left.+\frac{q-1}{T}\left(m_{T} \cosh y-\mu\right)\right]^{-q /(q-1)} d y .
\end{aligned}
$$

In the mid-rapidity $(y=0)$ region, the formulism of Tsallis statistics can be given by [31]

$$
f_{1}\left(p_{T}\right)=C_{T} p_{T} m_{T}\left[1+\frac{q-1}{T}\left(m_{T}-\mu\right)\right]^{-q /(q-1)},
$$

where $C_{T}=g V /(2 \pi)^{2}$ is the normalization constant related to the free parameters. When the collision energy is high enough, the chemical potential is especially small. In the energy range of LHC, the value of $\mu$ approximately is zero [27-29].

In some cases, the experimental data are presented in a given rapidity range, which is generally not in the midrapidity region. We have to shift simply the given rapidity range to the mid-rapidity region by subtracting the midvalue of the given rapidity range and use (3) directly. If we consider the differences of rapidity in the given rapidity range or in the mid-rapidity region, a more accurate equation (2) which includes the integral for the rapidity can be used. If we consider the given rapidity range in the more accurate equation (2), the kinetic energy of directional movement will be included in the temperature, which causes a larger temperature and is not correct. In fact, in the mid-rapidity region, the difference between the minimum (maximum) rapidity and 0 is neglected. The more accurate equation (2) is not needed.

It should be noted that when we use the multisource thermal model and the Tsallis statistics, each group or process is assumed to stay in a local equilibrium state. The excitation degree of each group or process is described by the temperature parameter $T$, and the equilibrium degree is described by the entropy index $q$. A large $T$ corresponds to a high excitation degree, and a large $q(q \gg 1)$ corresponds to a far away from the equilibrium state. The closer to 1 the $q$ is, the closer to equilibrium the group or process becomes. In an equilibrium state, one has $q=1$. Generally, $q$ is not too large. This means that each group or process stays approximately in a local equilibrium state.

The inverse power-law can describe the hard and veryhard processes. In [32-34], the inverse power-law is described by the Hagedorn function [35]; its parameterized form is expressed as

$$
f_{2}\left(p_{T}\right)=A p_{T}\left(1+\frac{p_{T}}{p_{0}}\right)^{-n}
$$

where $p_{0}$ and $n$ are free parameters and $A$ is the normalization constant related to the free parameters. 


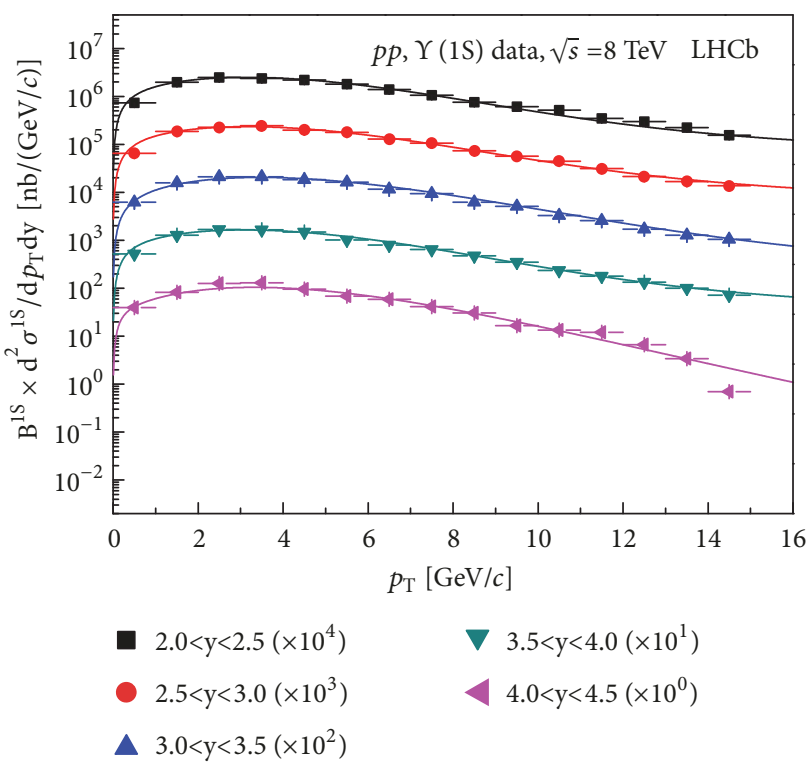

(a)

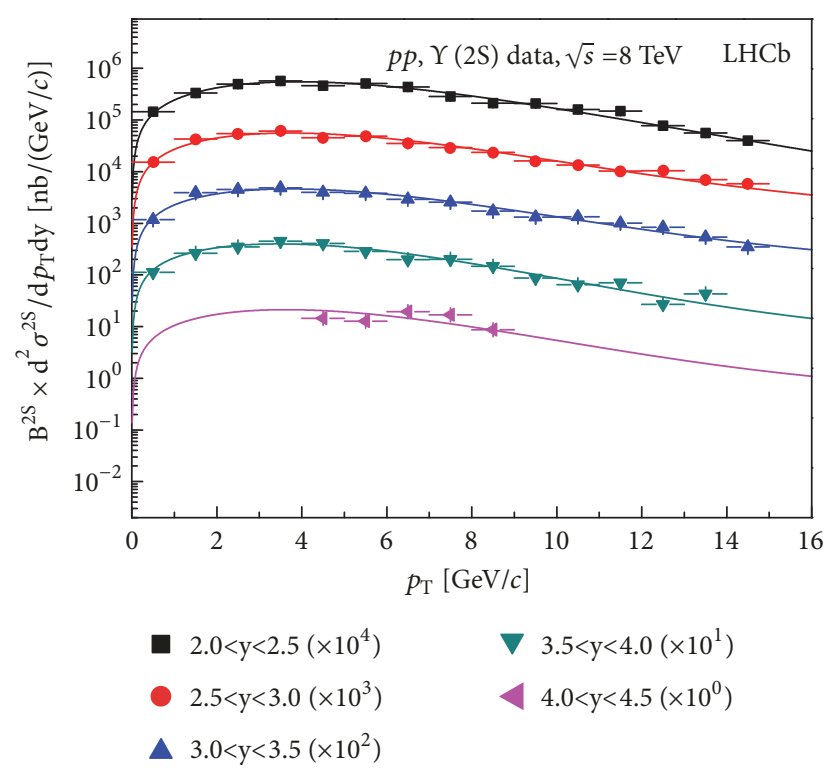

(b)

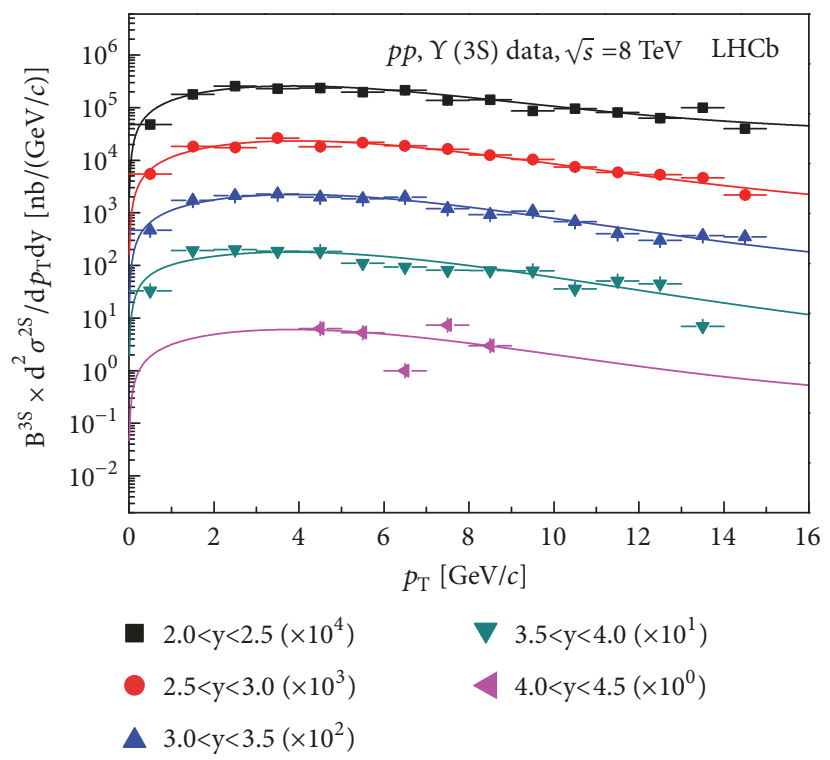

(c)

Figure 5: Transverse momentum spectra of $\Upsilon$ mesons $(\Upsilon(1 S), \Upsilon(2 S)$, and $\Upsilon(3 S)$ ) produced in $p p$ collision at $\sqrt{s}=8$ TeV. The symbols with the error bars represent the experimental data with the quadratic sums of the statistical and systematic uncertainties measured by the LHCb Collaboration [16]. The curves are our fitted results.

In the Hagedorn function, scattering between nucleons may be thought of in terms of valence quarks. To measure the scattering strength, the parameters $p_{0}$ and $n$ can be used. A large $p_{0}$ and a small $n$ describe a wide $p_{T}$ range which means a violent scattering. Impact between quarks may also be described via $\mathrm{pQCD}$ (perturbative quantum chromodynamics), which gives an inverse power-law $p_{T}$ spectrum [3234] which is the same as the Hagedorn function [35]. The $\mathrm{pQCD}$ also gives rapidity dependent $p_{T}$ spectra which results in rapidity dependent $p_{0}$ and $n$.
According to (3) and (4), we can structure a superposition of the Tsallis statistics and the inverse power-law, which results in a two-component statistical model as

$$
f\left(p_{T}\right)=k f_{1}\left(p_{T}\right)+(1-k) f_{2}\left(p_{T}\right),
$$

where $k$ is the contribution ratio of the first component. Naturally, (5) is normalized to 1 due to the fact that (3) and (4) are normalized to 1 . Although the Tsallis statistics has more than one forms and the inverse power-law has different modified 


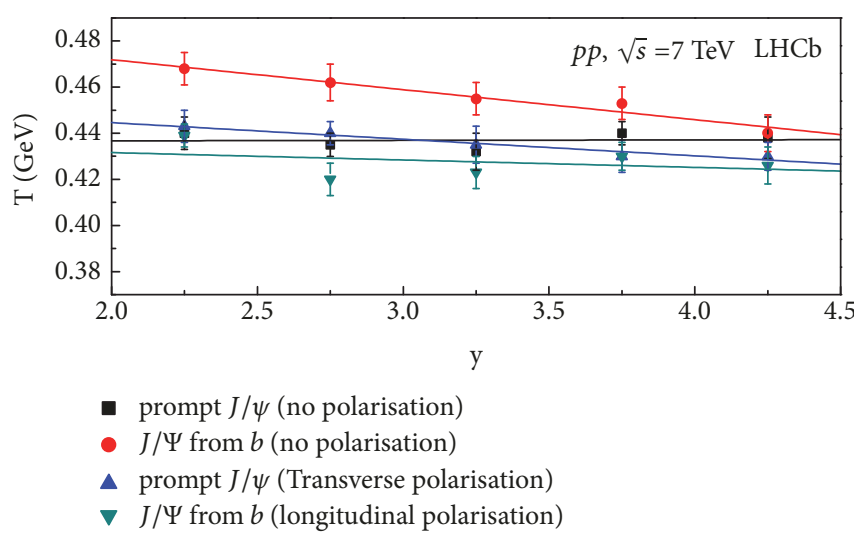

(a)

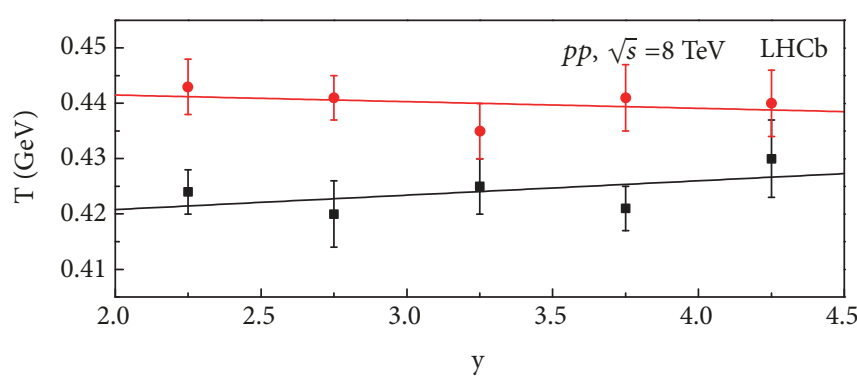

- prompt $J / \psi$

$J / \psi$ from $b$

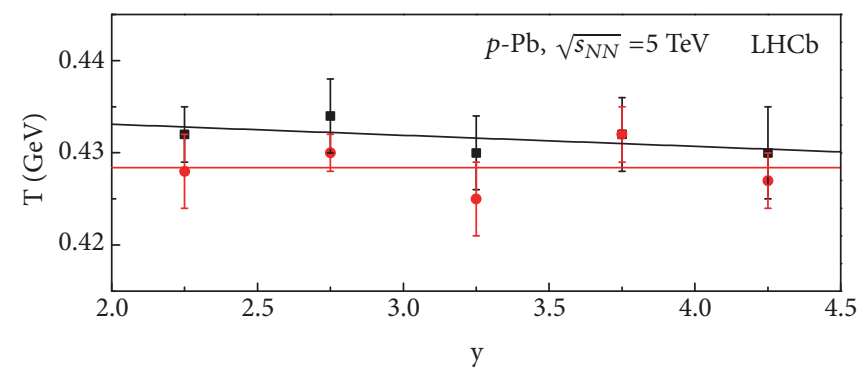

- $\operatorname{prompt} J / \psi$

- $J / \psi$ from $b$

(b)

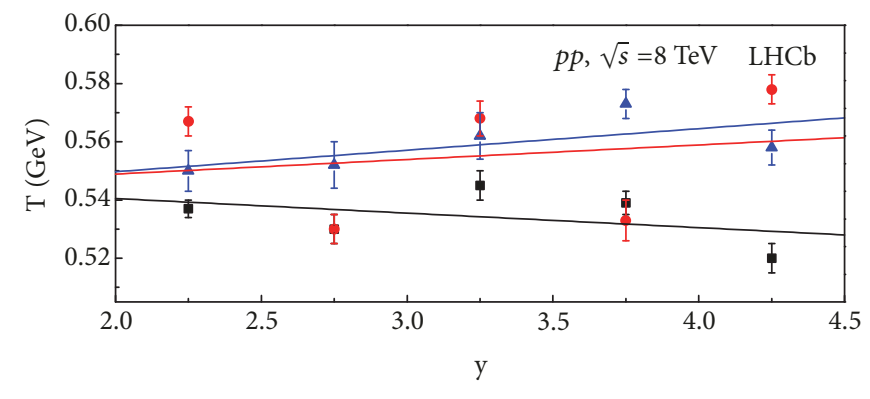

- $\Upsilon(1 S)$

- $\Upsilon(2 S$

- $\Upsilon(3 S)$

(c)

(d)

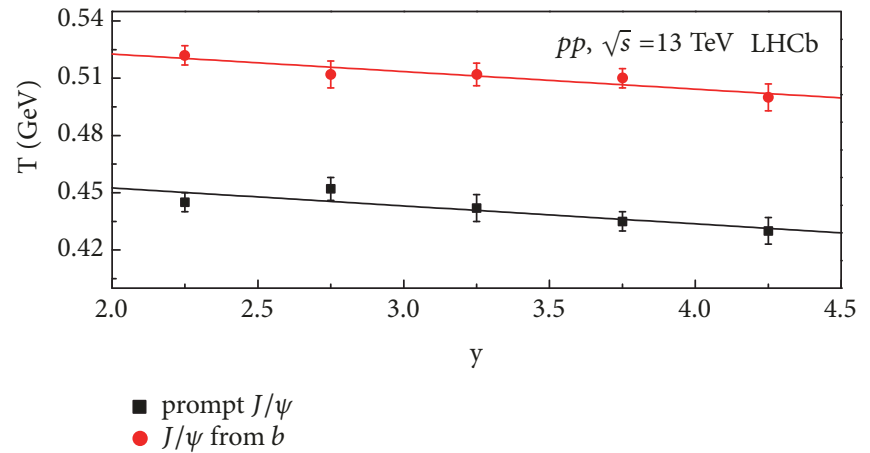

(e)

Figure 6: The relationship between $T$ and $y$ for $J / \psi$ and $\Upsilon$ mesons produced in $p p$ and $p$-Pb collisions at the LHC. The symbols are quoted in Table 1, and the lines are our fitted results.

forms, we shall not discuss them further. In fact, (5) structured through (3) and (4) is enough to use in the present work.

It should be noted that there are two types of superposition for two components. Except for (5), another superposition is the step function or the Hagedorn model [35]

$$
\begin{aligned}
f\left(p_{T}\right)= & A_{1} \theta\left(p_{1}-p_{T}\right) f_{1}\left(p_{T}\right) \\
& +A_{2} \theta\left(p_{T}-p_{1}\right) f_{2}\left(p_{T}\right),
\end{aligned}
$$

where $A_{1}$ and $A_{2}$ are constants which ensure the contributions of two components are the same at $p_{T}=p_{1}$, and $\theta(x)=1$ if $x>0$, and $\theta(x)=0$ if $x<0$. Although there are entanglements in determining parameters by (5), the curve at $p_{T}=p_{1}$ is not smooth due to (6). Our very recent work [36] shows that (5) and (6) result in similar values of parameters, especially for the trends. To obtain a smooth curve, (5) is used in the present work.

For a real fit process, we may select firstly a set of free parameters. Then, we may use the selected set of parameters in (3) and (4), and let the two equations be normalized to 1, respectively. The normalization constants $C_{T}$ and $A$ can be determined and used back in (3) and (4) so that the two equations can be used in (5). In the determination for the parameters, the method of least squares can be used. 


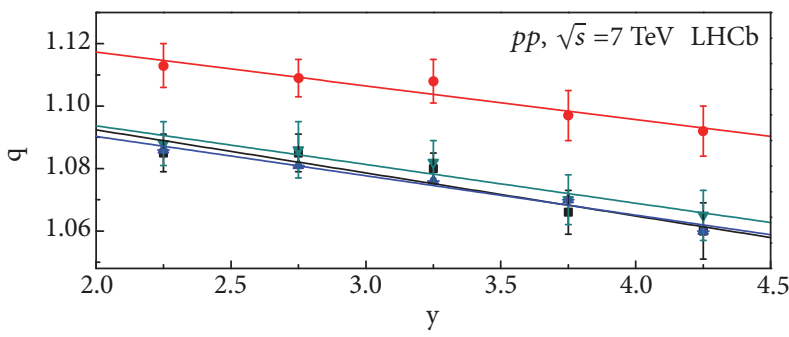

- prompt $J / \psi$ (no polarisation)

- $J / \psi$ from $b$ (no polarisation)

- prompt $J / \psi$ (Transverse polarisation)

$\nabla J / \psi$ from $b$ (longitudinal polarisation)

(a)

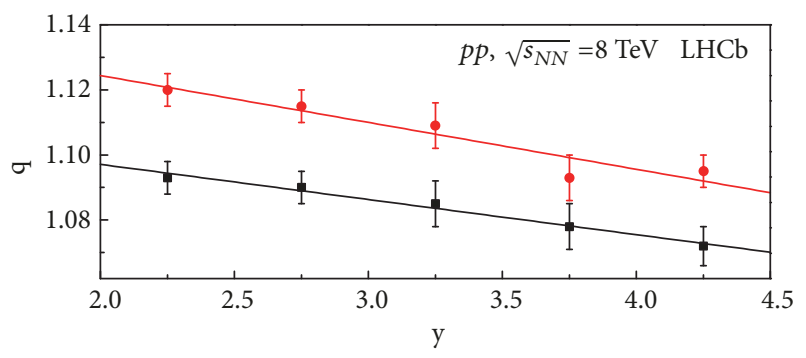

- prompt $J / \psi$

- $J / \psi$ from $b$

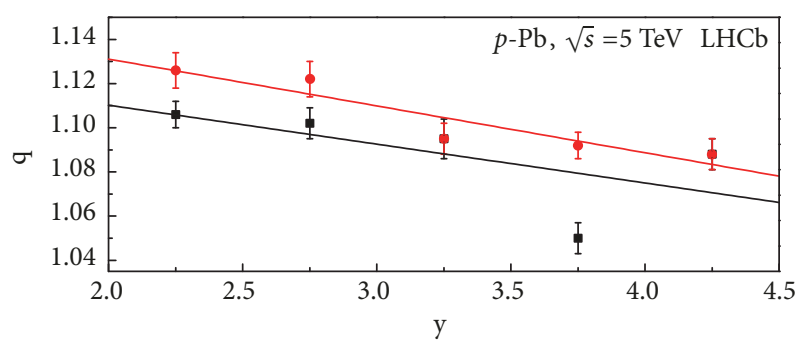

- prompt $J / \psi$

- $J / \psi$ from $b$

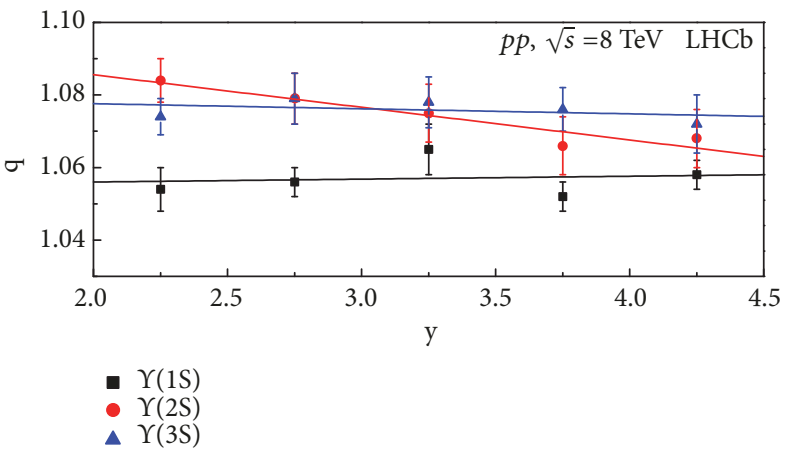

(d)

(c)

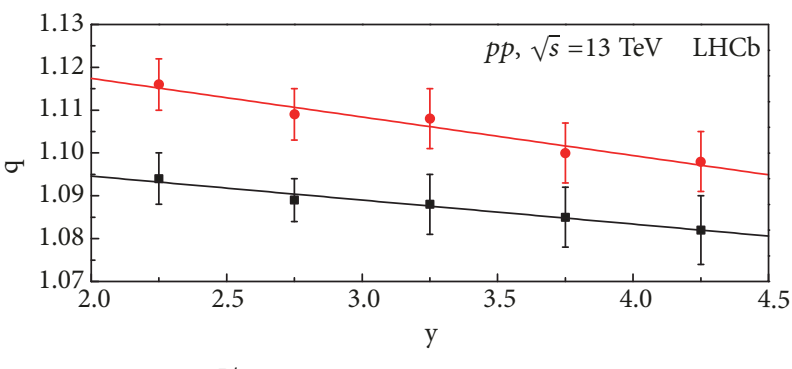

- prompt $J / \psi$

- $J / \psi$ from $b$

(e)

FIGURE 7: The same as Figure 6, but showing the relationship between $q$ and $y$.

The errors of the parameters can be determined to let the confidence levels of fittings be $95 \%$ in most cases and $90 \%$ in a few cases if existent.

\section{Results and Discussion}

Figure 1 shows the transverse momentum spectra, $d^{2} \sigma(J /$ $\psi) d p_{T} d y$, of $J / \psi$ mesons produced in $p p$ collisions at centerof-mass energy $\sqrt{s}=7 \mathrm{TeV}$, where $\sigma$ denotes the cross section. Figures $1(\mathrm{a})-1(\mathrm{~d})$ present the results of the prompt $J / \psi$ with no polarisation, $J / \psi$ from $b$ with no polarisation, prompt $J / \psi$ with full transverse polarisation, and prompt $J / \psi$ with full longitudinal polarisation, respectively. The symbols represent the experimental data measured by the $\mathrm{LHCb}$ Collaboration [15] at the LHC. In order to see clearly, different symbols are used to distinguish the different rapidity ranges in the panels. The curves are our results fitted by (5). The values of free parameters $\left(k, T, q, p_{0}\right.$, and $\left.n\right)$ and $\chi^{2} / d o f$ (degree of freedom) corresponding to each curve in Figure 1 are listed in Table 1, where the normalization constants which reflect the areas under the curves are not listed to avoid trivialness. For the same reason, the concrete confidence levels are not listed in the table one by one. One can see that the experimental data measured by the LHCb Collaboration are well fitted by the two-component statistical model. The behaviors of parameters will be discussed later.

Figures 2 and 3 show the transverse momentum spectra of $J / \psi$ mesons produced in $p p$ collisions at $\sqrt{s}=8$ and $13 \mathrm{TeV}$, respectively. Figures 2(a) and 2(b) (Figures 3(a) and $3(\mathrm{~b})$ ) present the results of the prompt $J / \psi$ and $J / \psi$ from $b$, respectively. The symbols represent the experimental data measured by the LHCb Collaboration $[16,18]$ at the LHC and 
TABLE 1: Values of parameters and $\chi^{2} / d o f$ corresponding to the curves in Figures 1-5.

\begin{tabular}{|c|c|c|c|c|c|c|c|}
\hline Figure & Type & $k$ & $T(\mathrm{GeV})$ & $q$ & $p_{0}(\mathrm{GeV} / c)$ & $n$ & $\chi^{2} / d o f$ \\
\hline \multirow{5}{*}{ Figure 1(a) } & $2.0<y<2.5$ & $0.890 \pm 0.010$ & $0.440 \pm 0.007$ & $1.085 \pm 0.006$ & $1.622 \pm 0.008$ & $5.000 \pm 0.020$ & 0.096 \\
\hline & $2.5<y<3.0$ & $0.900 \pm 0.005$ & $0.435 \pm 0.005$ & $1.085 \pm 0.006$ & $1.622 \pm 0.008$ & $5.050 \pm 0.017$ & 0.164 \\
\hline & $3.0<y<3.5$ & $0.888 \pm 0.003$ & $0.432 \pm 0.008$ & $1.080 \pm 0.005$ & $1.625 \pm 0.009$ & $5.056 \pm 0.024$ & 0.614 \\
\hline & $3.5<y<4.0$ & $0.895 \pm 0.005$ & $0.440 \pm 0.005$ & $1.066 \pm 0.007$ & $1.623 \pm 0.007$ & $5.050 \pm 0.018$ & 0.450 \\
\hline & $4.0<y<4.5$ & $0.865 \pm 0.007$ & $0.438 \pm 0.009$ & $1.060 \pm 0.009$ & $1.605 \pm 0.013$ & $5.031 \pm 0.019$ & 0.317 \\
\hline \multirow{5}{*}{ Figure 1(b) } & $2.0<y<2.5$ & $0.902 \pm 0.005$ & $0.468 \pm 0.007$ & $1.113 \pm 0.007$ & $1.627 \pm 0.017$ & $5.037 \pm 0.017$ & 0.533 \\
\hline & $2.5<y<3.0$ & $0.895 \pm 0.003$ & $0.462 \pm 0.008$ & $1.109 \pm 0.006$ & $1.606 \pm 0.009$ & $5.050 \pm 0.020$ & 0.296 \\
\hline & $3.0<y<3.5$ & $0.892 \pm 0.003$ & $0.455 \pm 0.007$ & $1.108 \pm 0.007$ & $1.600 \pm 0.010$ & $5.036 \pm 0.023$ & 0.414 \\
\hline & $3.5<y<4.0$ & $0.893 \pm 0.005$ & $0.453 \pm 0.007$ & $1.097 \pm 0.008$ & $1.603 \pm 0.010$ & $5.026 \pm 0.012$ & 0.226 \\
\hline & $4.0<y<4.5$ & $0.897 \pm 0.006$ & $0.440 \pm 0.008$ & $1.092 \pm 0.008$ & $1.588 \pm 0.018$ & $5.030 \pm 0.020$ & 0.276 \\
\hline \multirow{5}{*}{ Figure 1(c) } & $2.0<y<2.5$ & $0.900 \pm 0.006$ & $0.443 \pm 0.007$ & $1.086 \pm 0.008$ & $1.607 \pm 0.007$ & $5.000 \pm 0.015$ & 0.075 \\
\hline & $2.5<y<3.0$ & $0.896 \pm 0.003$ & $0.440 \pm 0.005$ & $1.081 \pm 0.006$ & $1.588 \pm 0.012$ & $5.000 \pm 0.017$ & 0.485 \\
\hline & $3.0<y<3.5$ & $0.894 \pm 0.008$ & $0.435 \pm 0.008$ & $1.076 \pm 0.008$ & $1.600 \pm 0.005$ & $5.000 \pm 0.020$ & 0.392 \\
\hline & $3.5<y<4.0$ & $0.892 \pm 0.006$ & $0.430 \pm 0.007$ & $1.070 \pm 0.005$ & $1.603 \pm 0.005$ & $5.008 \pm 0.018$ & 0.397 \\
\hline & $4.0<y<4.5$ & $0.875 \pm 0.008$ & $0.430 \pm 0.006$ & $1.060 \pm 0.009$ & $1.588 \pm 0.010$ & $5.003 \pm 0.013$ & 0.150 \\
\hline \multirow{5}{*}{ Figure 1(d) } & $2.0<y<2.5$ & $0.896 \pm 0.004$ & $0.439 \pm 0.005$ & $1.088 \pm 0.007$ & $1.602 \pm 0.008$ & $5.005 \pm 0.018$ & 0.557 \\
\hline & $2.5<y<3.0$ & $0.899 \pm 0.008$ & $0.420 \pm 0.007$ & $1.086 \pm 0.009$ & $1.582 \pm 0.012$ & $5.010 \pm 0.015$ & 0.324 \\
\hline & $3.0<y<3.5$ & $0.895 \pm 0.007$ & $0.423 \pm 0.007$ & $1.082 \pm 0.007$ & $1.612 \pm 0.012$ & $5.000 \pm 0.015$ & 0.438 \\
\hline & $3.5<y<4.0$ & $0.893 \pm 0.008$ & $0.430 \pm 0.006$ & $1.070 \pm 0.008$ & $1.608 \pm 0.007$ & $5.008 \pm 0.017$ & 0.517 \\
\hline & $4.0<y<4.5$ & $0.885 \pm 0.005$ & $0.426 \pm 0.008$ & $1.065 \pm 0.008$ & $1.583 \pm 0.010$ & $5.012 \pm 0.013$ & 0.330 \\
\hline \multirow{5}{*}{ Figure 2(a) } & $2.0<y<2.5$ & $0.896 \pm 0.002$ & $0.424 \pm 0.004$ & $1.093 \pm 0.005$ & $1.605 \pm 0.008$ & $5.000 \pm 0.020$ & 0.258 \\
\hline & $2.5<y<3.0$ & $0.886 \pm 0.004$ & $0.420 \pm 0.006$ & $1.090 \pm 0.005$ & $1.612 \pm 0.005$ & $5.003 \pm 0.015$ & 0.245 \\
\hline & $3.0<y<3.5$ & $0.890 \pm 0.005$ & $0.425 \pm 0.005$ & $1.085 \pm 0.007$ & $1.603 \pm 0.005$ & $5.007 \pm 0.013$ & 0.179 \\
\hline & $3.5<y<4.0$ & $0.886 \pm 0.004$ & $0.421 \pm 0.004$ & $1.078 \pm 0.007$ & $1.600 \pm 0.005$ & $5.012 \pm 0.016$ & 0.413 \\
\hline & $4.0<y<4.5$ & $0.889 \pm 0.003$ & $0.430 \pm 0.007$ & $1.072 \pm 0.006$ & $1.600 \pm 0.007$ & $5.022 \pm 0.016$ & 0.899 \\
\hline \multirow{5}{*}{ Figure 2(b) } & $2.0<y<2.5$ & $0.870 \pm 0.004$ & $0.443 \pm 0.005$ & $1.120 \pm 0.005$ & $1.627 \pm 0.007$ & $4.988 \pm 0.018$ & 2.087 \\
\hline & $2.5<y<3.0$ & $0.852 \pm 0.003$ & $0.441 \pm 0.004$ & $1.115 \pm 0.005$ & $1.622 \pm 0.005$ & $4.992 \pm 0.016$ & 2.057 \\
\hline & $3.0<y<3.5$ & $0.850 \pm 0.003$ & $0.435 \pm 0.005$ & $1.109 \pm 0.007$ & $1.623 \pm 0.008$ & $4.825 \pm 0.015$ & 1.321 \\
\hline & $3.5<y<4.0$ & $0.802 \pm 0.004$ & $0.441 \pm 0.006$ & $1.093 \pm 0.007$ & $1.613 \pm 0.008$ & $4.867 \pm 0.017$ & 2.980 \\
\hline & $4.0<y<4.5$ & $0.847 \pm 0.003$ & $0.440 \pm 0.006$ & $1.095 \pm 0.005$ & $1.605 \pm 0.010$ & $5.000 \pm 0.021$ & 0.902 \\
\hline \multirow{5}{*}{ Figure 3(a) } & $2.0<y<2.5$ & $0.860 \pm 0.004$ & $0.445 \pm 0.005$ & $1.094 \pm 0.006$ & $1.626 \pm 0.006$ & $4.095 \pm 0.023$ & 1.221 \\
\hline & $2.5<y<3.0$ & $0.867 \pm 0.003$ & $0.452 \pm 0.006$ & $1.089 \pm 0.005$ & $1.650 \pm 0.008$ & $4.452 \pm 0.018$ & 1.569 \\
\hline & $3.0<y<3.5$ & $0.868 \pm 0.005$ & $0.442 \pm 0.007$ & $1.088 \pm 0.007$ & $1.635 \pm 0.007$ & $4.380 \pm 0.020$ & 1.937 \\
\hline & $3.5<y<4.0$ & $0.865 \pm 0.003$ & $0.435 \pm 0.005$ & $1.085 \pm 0.007$ & $1.630 \pm 0.008$ & $4.630 \pm 0.024$ & 1.070 \\
\hline & $4.0<y<4.5$ & $0.858 \pm 0.003$ & $0.430 \pm 0.007$ & $1.082 \pm 0.008$ & $1.608 \pm 0.010$ & $4.672 \pm 0.026$ & 1.062 \\
\hline \multirow{5}{*}{ Figure 3(b) } & $2.0<y<2.5$ & $0.886 \pm 0.004$ & $0.522 \pm 0.005$ & $1.116 \pm 0.006$ & $1.635 \pm 0.005$ & $3.150 \pm 0.015$ & 0.061 \\
\hline & $2.5<y<3.0$ & $0.867 \pm 0.005$ & $0.512 \pm 0.007$ & $1.109 \pm 0.006$ & $1.653 \pm 0.008$ & $3.352 \pm 0.017$ & 0.886 \\
\hline & $3.0<y<3.5$ & $0.887 \pm 0.003$ & $0.512 \pm 0.006$ & $1.108 \pm 0.007$ & $1.644 \pm 0.007$ & $3.365 \pm 0.017$ & 0.308 \\
\hline & $3.5<y<4.0$ & $0.865 \pm 0.005$ & $0.510 \pm 0.005$ & $1.100 \pm 0.007$ & $1.641 \pm 0.007$ & $3.640 \pm 0.020$ & 0.464 \\
\hline & $4.0<y<4.5$ & $0.885 \pm 0.006$ & $0.500 \pm 0.007$ & $1.098 \pm 0.007$ & $1.628 \pm 0.008$ & $3.972 \pm 0.020$ & 0.638 \\
\hline \multirow{5}{*}{ Figure 4(a) } & $2.0<y<2.5$ & $0.880 \pm 0.003$ & $0.432 \pm 0.003$ & $1.106 \pm 0.006$ & $1.607 \pm 0.005$ & $5.012 \pm 0.023$ & 1.372 \\
\hline & $2.5<y<3.0$ & $0.878 \pm 0.004$ & $0.434 \pm 0.004$ & $1.102 \pm 0.007$ & $1.608 \pm 0.007$ & $4.998 \pm 0.025$ & 1.788 \\
\hline & $3.0<y<3.5$ & $0.883 \pm 0.003$ & $0.430 \pm 0.004$ & $1.095 \pm 0.009$ & $1.610 \pm 0.005$ & $4.995 \pm 0.025$ & 1.816 \\
\hline & $3.5<y<4.0$ & $0.884 \pm 0.005$ & $0.432 \pm 0.004$ & $1.085 \pm 0.007$ & $1.608 \pm 0.006$ & $4.990 \pm 0.020$ & 2.044 \\
\hline & $4.0<y<4.5$ & $0.882 \pm 0.005$ & $0.430 \pm 0.005$ & $1.088 \pm 0.007$ & $1.607 \pm 0.008$ & $5.010 \pm 0.027$ & 1.639 \\
\hline \multirow{5}{*}{ Figure 4(b) } & $2.0<y<2.5$ & $0.858 \pm 0.003$ & $0.428 \pm 0.004$ & $1.126 \pm 0.008$ & $1.623 \pm 0.008$ & $3.956 \pm 0.024$ & 0.715 \\
\hline & $2.5<y<3.0$ & $0.839 \pm 0.003$ & $0.430 \pm 0.002$ & $1.122 \pm 0.008$ & $1.608 \pm 0.007$ & $3.998 \pm 0.027$ & 0.739 \\
\hline & $3.0<y<3.5$ & $0.705 \pm 0.004$ & $0.425 \pm 0.004$ & $1.095 \pm 0.007$ & $1.601 \pm 0.005$ & $3.977 \pm 0.023$ & 3.504 \\
\hline & $3.5<y<4.0$ & $0.717 \pm 0.003$ & $0.432 \pm 0.003$ & $1.092 \pm 0.006$ & $1.603 \pm 0.007$ & $3.890 \pm 0.030$ & 6.600 \\
\hline & $4.0<y<4.5$ & $0.715 \pm 0.005$ & $0.427 \pm 0.003$ & $1.088 \pm 0.007$ & $1.603 \pm 0.006$ & $4.010 \pm 0.030$ & 6.856 \\
\hline
\end{tabular}


TABle 1: Continued.

\begin{tabular}{lccccccc}
\hline Figure & Type & $k$ & $T(\mathrm{GeV})$ & $q$ & $p_{0}(\mathrm{GeV} / \mathrm{c})$ & $n$ & $\chi^{2} / d o f$ \\
\hline & $2.0<y<2.5$ & $0.776 \pm 0.002$ & $0.537 \pm 0.003$ & $1.054 \pm 0.006$ & $1.556 \pm 0.013$ & $2.355 \pm 0.010$ \\
& $2.5<y<3.0$ & $0.780 \pm 0.003$ & $0.530 \pm 0.005$ & $1.056 \pm 0.004$ & $1.568 \pm 0.018$ & $2.338 \pm 0.012$ & 0.490 \\
Figure 5(a) & $3.0<y<3.5$ & $0.865 \pm 0.005$ & $0.545 \pm 0.005$ & $1.065 \pm 0.007$ & $1.541 \pm 0.008$ & $2.372 \pm 0.012$ & 0.282 \\
& $3.5<y<4.0$ & $0.776 \pm 0.003$ & $0.539 \pm 0.004$ & $1.052 \pm 0.004$ & $1.563 \pm 0.012$ & $2.490 \pm 0.015$ & 0.503 \\
& $4.0<y<4.5$ & $0.903 \pm 0.005$ & $0.520 \pm 0.005$ & $1.058 \pm 0.004$ & $1.433 \pm 0.023$ & $4.052 \pm 0.022$ & 3.266 \\
\hline & $2.0<y<2.5$ & $0.912 \pm 0.004$ & $0.567 \pm 0.005$ & $1.084 \pm 0.006$ & $1.756 \pm 0.009$ & $2.655 \pm 0.015$ & 0.855 \\
& $2.5<y<3.0$ & $0.890 \pm 0.003$ & $0.530 \pm 0.005$ & $1.079 \pm 0.007$ & $1.791 \pm 0.012$ & $2.000 \pm 0.015$ & 0.921 \\
Figure 5(b) & $3.0<y<3.5$ & $0.870 \pm 0.003$ & $0.568 \pm 0.006$ & $1.075 \pm 0.008$ & $1.815 \pm 0.018$ & $2.000 \pm 0.013$ & 0.976 \\
& $3.5<y<4.0$ & $0.907 \pm 0.005$ & $0.533 \pm 0.007$ & $1.066 \pm 0.008$ & $1.708 \pm 0.025$ & $2.430 \pm 0.017$ & 1.801 \\
& $4.0<y<4.5$ & $0.896 \pm 0.003$ & $0.578 \pm 0.005$ & $1.068 \pm 0.008$ & $1.733 \pm 0.010$ & $2.052 \pm 0.010$ & 1.219 \\
\hline & $2.0<y<2.5$ & $0.705 \pm 0.003$ & $0.550 \pm 0.007$ & $1.074 \pm 0.005$ & $1.726 \pm 0.018$ & $1.705 \pm 0.017$ & 2.793 \\
& $2.5<y<3.0$ & $0.716 \pm 0.002$ & $0.552 \pm 0.008$ & $1.079 \pm 0.007$ & $1.788 \pm 0.024$ & $2.000 \pm 0.010$ & 1.146 \\
Figure 5(c) & $3.0<y<3.5$ & $0.725 \pm 0.005$ & $0.562 \pm 0.008$ & $1.078 \pm 0.007$ & $1.755 \pm 0.015$ & $2.185 \pm 0.015$ & 2.025 \\
& $3.5<y<4.0$ & $0.750 \pm 0.003$ & $0.573 \pm 0.005$ & $1.076 \pm 0.006$ & $1.738 \pm 0.018$ & $2.630 \pm 0.020$ & 7.854 \\
& $4.0<y<4.5$ & $0.736 \pm 0.005$ & $0.558 \pm 0.006$ & $1.072 \pm 0.008$ & $1.710 \pm 0.025$ & $2.052 \pm 0.012$ & 6.918 \\
\hline
\end{tabular}

the curves are our fitted results. The values of free parameters and $\chi^{2} /$ dof corresponding to each curve in Figures 2 and 3 are listed in Table 1, which will be discussed later. One can see again that the experimental data measured by the $\mathrm{LHCb}$ Collaboration are well fitted by the two-component statistical model.

The transverse momentum spectra of $J / \psi$ mesons produced in $p$ - $\mathrm{Pb}$ collisions at center-of-mass energy per nucleon pair $\sqrt{s_{\mathrm{NN}}}=5 \mathrm{TeV}$ are displayed in Figure 4. Figures 4(a) and 4 (b) present the results of the prompt $J / \psi$ and $J / \psi$ from $b$, respectively. The symbols represent the experimental data measured by the LHCb Collaboration [17] at the LHC and the curves are our fitted results. The values of free parameters and $\chi^{2} /$ dof corresponding to each curve in Figure 4 are listed in Table 1, which will be discussed later. Once again, the experimental data measured by the LHCb Collaboration are well fitted by the model.

The transverse momentum spectra of $\Upsilon$ mesons $(\Upsilon(1 S)$, $\Upsilon(2 S)$, and $\Upsilon(3 S)$ ) produced in $p p$ collisions at $\sqrt{s}=8 \mathrm{TeV}$ are shown in Figure 5 , where $B^{1 S}\left(B^{2 S}\right.$ and $\left.B^{3 S}\right)$ on the vertical axis denotes the branch ratio. The symbols represent the experimental data measured by the LHCb Collaboration [16] and the curves are our fitted results. The values of free parameters and $\chi^{2} /$ dof corresponding to each curve in Figure 5 are listed in Table 1. Once more, the experimental data measured by the LHCb Collaboration are well fitted by the model.

To see clearly the relationships between the free parameters $\left(T, q, p_{0}\right.$, and $\left.n\right)$ and rapidity, we plot the parameter values listed in Table 1 in Figures 6-9, respectively. In the four figures, the symbols represent the parameters and the lines are our fitted results, though some of them do not obey the linear functions. In Figure 9, some error bars are smaller than the symbol size due to wide coordinate range. The intercepts, slopes, and $\chi^{2} /$ dof corresponding to the lines in Figures 6-9 are listed in Table 2 . One can see that, in the error range, the parameter $T$ does not show an obvious change or has a slight decrease in most cases, the parameters $q$ and $p_{0}$ appear to decrease, and the parameter $n$ does not show an obvious change or has a slight increase, with the increase of rapidity.

It should be noted that Figure 6(d) shows a slight increase of $T$ for $\Upsilon(2 S)$ and $\Upsilon(3 S)$, and a slight decrease of $T$ for $\Upsilon(1 S)$, with the increase of rapidity. An average weighted by different yields will result in a slight decrease of $T$ with the increase of rapidity. The rapidity dependent $p_{0}$ and $n$ confirm the prediction of pQCD which gives the inverse power-law spectra being rapidity dependent [32-34].

The meanings of parameters can be explained by us. The invariant or slight decreasing temperature parameter renders that the excitation degree of the interacting system keeps invariant or slight decreasing trend with the increase of rapidity. The temperature is not the "real" temperature at the stage of kinetic freeze-out, but the effective temperature in which the contribution of flow effect is not excluded. Even the flow effect is excluding, the kinetic freeze-out temperature from the spectra of heavy quarkonia is much higher than that from the spectra of light particles. This means that the heavy quarkonia produce much earlier than light particles in the collision process.

All values of the entropy index are close to 1 , which means that the interacting system stays approximately at the (local) equilibrium state, even if in small collision systems such as $p p$ and $p-\mathrm{Pb}$ collisions. The decreasing entropy index renders that the interacting system reaches a more equilibrium state in the very forward rapidity region. We believe that the interacting system stays at the (local) equilibrium state in large collision systems such as lead-lead and other nucleusnucleus collisions.

The decreasing $p_{0}$ and increasing $n$ render a narrow $p_{T}$ range. The present work shows a slightly narrow $p_{T}$ range in the very forward rapidity region. This means that the scattering strength of the interacting system decreases slightly with the increase of rapidity. This observation confirms the result from the temperature parameter. 
TABLE 2: Values of intercepts, slopes, and $\chi^{2} /$ dof corresponding to the lines in Figures 6-9.

\begin{tabular}{|c|c|c|c|c|}
\hline Figure & Type & Intercept & Slope & $\chi^{2} / d o f$ \\
\hline \multirow{4}{*}{ Figure 6(a) } & prompt $J / \psi$ (no polarisation) & $0.436 \pm 0.007$ & $0.000 \pm 0.002$ & 0.363 \\
\hline & $J / \psi$ from $b$ (no polarisation) & $0.498 \pm 0.005$ & $-0.013 \pm 0.002$ & 0.144 \\
\hline & prompt $J / \psi$ (transverse polarisation) & $0.459 \pm 0.003$ & $-0.007 \pm 0.001$ & 0.062 \\
\hline & $J / \psi$ from $b$ (longitudinal polarisation) & $0.438 \pm 0.015$ & $-0.003 \pm 0.004$ & 1.778 \\
\hline \multirow{2}{*}{ Figure 6(b) } & prompt $J / \psi$ & $0.436 \pm 0.003$ & $-0.001 \pm 0.001$ & 0.168 \\
\hline & $J / \psi$ from $b$ & $0.428 \pm 0.006$ & $0.000 \pm 0.002$ & 1.010 \\
\hline \multirow{2}{*}{ Figure 6(c) } & prompt $J / \psi$ & $0.416 \pm 0.007$ & $0.003 \pm 0.002$ & 0.686 \\
\hline & $J / \psi$ from $b$ & $0.444 \pm 0.006$ & $-0.001 \pm 0.002$ & 0.417 \\
\hline \multirow{3}{*}{ Figure 6(d) } & $\Upsilon(1 S)$ & $0.550 \pm 0.018$ & $-0.005 \pm 0.006$ & 4.572 \\
\hline & $\Upsilon(2 S)$ & $0.539 \pm 0.046$ & $0.005 \pm 0.014$ & 20.526 \\
\hline & $\Upsilon(3 S)$ & $0.535 \pm 0.015$ & $0.007 \pm 0.004$ & 2.189 \\
\hline \multirow{2}{*}{ Figure 6(e) } & prompt $J / \psi$ & $0.471 \pm 0.009$ & $-0.009 \pm 0.003$ & 0.791 \\
\hline & $J / \psi$ from $b$ & $0.541 \pm 0.006$ & $-0.009 \pm 0.002$ & 0.318 \\
\hline \multirow{4}{*}{ Figure 7(a) } & prompt $J / \psi$ (no polarisation) & $1.120 \pm 0.008$ & $-0.014 \pm 0.002$ & 0.338 \\
\hline & $J / \psi$ from $b$ (no polarisation) & $1.139 \pm 0.005$ & $-0.011 \pm 0.002$ & 0.153 \\
\hline & prompt $J / \psi$ (transverse polarisation) & $1.116 \pm 0.003$ & $-0.013 \pm 0.001$ & 0.073 \\
\hline & $J / \psi$ from $\mathrm{b}$ (longitudinal polarisation) & $1.118 \pm 0.006$ & $-0.012 \pm 0.002$ & 0.179 \\
\hline \multirow{2}{*}{ Figure 7(b) } & prompt $J / \psi$ & $1.145 \pm 0.037$ & $-0.018 \pm 0.011$ & 8.300 \\
\hline & $J / \psi$ from $b$ & $1.174 \pm 0.014$ & $-0.021 \pm 0.004$ & 1.049 \\
\hline \multirow{2}{*}{ Figure 7(c) } & prompt $J / \psi$ & $1.119 \pm 0.002$ & $-0.011 \pm 0.001$ & 0.059 \\
\hline & $J / \psi$ from $b$ & $1.153 \pm 0.008$ & $-0.014 \pm 0.002$ & 0.462 \\
\hline \multirow{3}{*}{ Figure $7(d)$} & $\Upsilon(1 S)$ & $1.054 \pm 0.010$ & $0.001 \pm 0.003$ & 1.096 \\
\hline & $\Upsilon(2 S)$ & $1.104 \pm 0.005$ & $-0.009 \pm 0.002$ & 0.120 \\
\hline & $\Upsilon(3 S)$ & $1.080 \pm 0.006$ & $-0.001 \pm 0.002$ & 0.250 \\
\hline \multirow{2}{*}{ Figure 7(e) } & prompt $J / \psi$ & $1.106 \pm 0.002$ & $-0.006 \pm 0.001$ & 0.033 \\
\hline & $J / \psi$ from $b$ & $1.135 \pm 0.003$ & $-0.009 \pm 0.001$ & 0.079 \\
\hline \multirow{4}{*}{ Figure 8(a) } & prompt $J / \psi$ (no polarisation) & $1.641 \pm 0.013$ & $-0.007 \pm 0.004$ & 0.659 \\
\hline & $J / \psi$ from $b$ (no polarisation) & $1.657 \pm 0.013$ & $-0.016 \pm 0.004$ & 0.447 \\
\hline & prompt $J / \psi$ (transverse polarisation) & $1.612 \pm 0.017$ & $-0.005 \pm 0.005$ & 1.540 \\
\hline & $J / \psi$ from $b$ (longitudinal polarisation) & $1.605 \pm 0.029$ & $-0.002 \pm 0.009$ & 2.584 \\
\hline \multirow{2}{*}{ Figure 8(b) } & prompt $J / \psi$ & $1.608 \pm 0.003$ & $0.000 \pm 0.001$ & 0.072 \\
\hline & $J / \psi$ from $b$ & $1.637 \pm 0.012$ & $-0.009 \pm 0.004$ & 1.088 \\
\hline \multirow{2}{*}{ Figure $8(c)$} & prompt $J / \psi$ & $1.618 \pm 0.007$ & $-0.004 \pm 0.002$ & 0.566 \\
\hline & $J / \psi$ from $b$ & $1.652 \pm 0.006$ & $-0.011 \pm 0.002$ & 0.190 \\
\hline \multirow{3}{*}{ Figure $8(d)$} & $\Upsilon(1 S)$ & $1.695 \pm 0.084$ & $-0.050 \pm 0.025$ & 10.642 \\
\hline & $\Upsilon(2 S)$ & $1.844 \pm 0.080$ & $-0.026 \pm 0.024$ & 8.408 \\
\hline & $\Upsilon(3 S)$ & $1.797 \pm 0.057$ & $-0.016 \pm 0.017$ & 2.304 \\
\hline \multirow{2}{*}{ Figure 8(e) } & prompt $J / \psi$ & $1.666 \pm 0.026$ & $-0.011 \pm 0.008$ & 3.927 \\
\hline & $J / \psi$ from $b$ & $1.657 \pm 0.018$ & $-0.005 \pm 0.005$ & 2.416 \\
\hline \multirow{4}{*}{ Figure 9(a) } & prompt $J / \psi$ (no polarisation) & $4.997 \pm 0.044$ & $0.012 \pm 0.013$ & 1.497 \\
\hline & $J / \psi$ from $b$ (no polarisation) & $5.060 \pm 0.014$ & $-0.008 \pm 0.004$ & 0.223 \\
\hline & prompt $J / \psi$ (transverse polarisation) & $4.993 \pm 0.006$ & $0.003 \pm 0.002$ & 0.033 \\
\hline & $J / \psi$ from $b$ (longitudinal polarisation) & $4.999 \pm 0.009$ & $0.002 \pm 0.003$ & 0.112 \\
\hline \multirow{2}{*}{ Figure 9(b) } & prompt $J / \psi$ & $5.009 \pm 0.020$ & $-0.002 \pm 0.006$ & 0.215 \\
\hline & $J / \psi$ from $b$ & $3.966 \pm 0.099$ & $0.000 \pm 0.030$ & 3.457 \\
\hline \multirow{2}{*}{ Figure 9(c) } & prompt $J / \psi$ & $4.974 \pm 0.004$ & $0.011 \pm 0.001$ & 0.024 \\
\hline & $J / \psi$ from $b$ & $5.000 \pm 0.170$ & $-0.020 \pm 0.051$ & 31.168 \\
\hline \multirow{3}{*}{ Figure 9(d) } & $\Upsilon(1 S)$ & $0.416 \pm 1.036$ & $0.709 \pm 0.311$ & 1450.893 \\
\hline & $\Upsilon(2 S)$ & $2.732 \pm 0.574$ & $-0.155 \pm 0.173$ & 441.653 \\
\hline & $\Upsilon(3 S)$ & $1.254 \pm 0.557$ & $0.265 \pm 0.167$ & 402.772 \\
\hline
\end{tabular}


TABLE 2: Continued.

\begin{tabular}{|c|c|c|c|c|}
\hline Figure & Type & Intercept & Slope & $\chi^{2} / d o f$ \\
\hline \multirow{2}{*}{ Figure 9(e) } & prompt $J / \psi$ & $3.580 \pm 0.197$ & $0.266 \pm 0.059$ & 30.391 \\
\hline & $J / \psi$ from $b$ & $2.240 \pm 0.187$ & $0.386 \pm 0.056$ & 33.711 \\
\hline \multirow{4}{*}{ Figure 10(a) } & prompt $J / \psi$ (no polarisation) & $2.960 \pm 0.110$ & $-0.161 \pm 0.033$ & 0.144 \\
\hline & $J / \psi$ from $b$ (no polarisation) & $3.692 \pm 0.098$ & $-0.231 \pm 0.030$ & 2.259 \\
\hline & prompt $J / \psi$ (transverse polarisation) & $3.038 \pm 0.071$ & $-0.191 \pm 0.021$ & 0.079 \\
\hline & $J / \psi$ from $b$ (longitudinal polarisation) & $2.855 \pm 0.086$ & $-0.127 \pm 0.026$ & 0.076 \\
\hline \multirow{2}{*}{ Figure 10(b) } & prompt $J / \psi$ & $3.273 \pm 0.087$ & $-0.170 \pm 0.026$ & 3.307 \\
\hline & $J / \psi$ from $b$ & $3.867 \pm 0.190$ & $-0.290 \pm 0.057$ & 18.317 \\
\hline \multirow{2}{*}{ Figure $10(c)$} & prompt $J / \psi$ & $3.031 \pm 0.060$ & $-0.157 \pm 0.018$ & 0.031 \\
\hline & $J / \psi$ from $b$ & $3.669 \pm 0.099$ & $-0.221 \pm 0.030$ & 4.616 \\
\hline \multirow{3}{*}{ Figure 10(d) } & $\Upsilon(1 S)$ & $5.429 \pm 0.278$ & $-0.155 \pm 0.083$ & 0.029 \\
\hline & $\Upsilon(2 S)$ & $5.529 \pm 0.697$ & $0.086 \pm 0.021$ & 0.025 \\
\hline & $\Upsilon(3 S)$ & $6.730 \pm 0.254$ & $-0.252 \pm 0.076$ & 0.015 \\
\hline \multirow{2}{*}{ Figure 10(e) } & prompt $J / \psi$ & $3.126 \pm 0.026$ & $-0.134 \pm 0.008$ & 0.002 \\
\hline & $J / \psi$ from $b$ & $3.995 \pm 0.070$ & $-0.217 \pm 0.021$ & 0.048 \\
\hline \multirow{4}{*}{ Figure 11(a) } & prompt $J / \psi$ (no polarisation) & $2.597 \pm 0.082$ & $-0.151 \pm 0.025$ & 0.020 \\
\hline & $J / \psi$ from $b$ (no polarisation) & $3.198 \pm 0.078$ & $-0.200 \pm 0.024$ & 0.076 \\
\hline & prompt $J / \psi$ (transverse polarisation) & $2.551 \pm 0.178$ & $-0.129 \pm 0.054$ & 0.201 \\
\hline & $J / \psi$ from $b$ (longitudinal polarisation) & $2.543 \pm 0.059$ & $-0.131 \pm 0.018$ & 0.011 \\
\hline \multirow{2}{*}{ Figure 11(b) } & prompt $J / \psi$ & $2.847 \pm 0.080$ & $-0.151 \pm 0.024$ & 0.149 \\
\hline & $J / \psi$ from $b$ & $3.379 \pm 0.163$ & $-0.249 \pm 0.049$ & 5.393 \\
\hline \multirow{2}{*}{ Figure 11(c) } & prompt $J / \psi$ & $2.620 \pm 0.040$ & $-0.135 \pm 0.012$ & 0.005 \\
\hline & $J / \psi$ from $b$ & $3.215 \pm 0.094$ & $-0.204 \pm 0.028$ & 0.195 \\
\hline \multirow{3}{*}{ Figure 11(d) } & $\Upsilon(1 S)$ & $4.537 \pm 0.238$ & $-0.134 \pm 0.072$ & 0.039 \\
\hline & $\Upsilon(2 S)$ & $5.039 \pm 0.273$ & $-0.115 \pm 0.082$ & 0.015 \\
\hline & $\Upsilon(3 S)$ & $5.527 \pm 0.223$ & $-0.205 \pm 0.067$ & 0.023 \\
\hline \multirow{2}{*}{ Figure 11(e) } & prompt $J / \psi$ & $2.574 \pm 0.264$ & $-0.057 \pm 0.079$ & 0.138 \\
\hline & $J / \psi$ from $b$ & $3.513 \pm 0.064$ & $-0.201 \pm 0.019$ & 0.014 \\
\hline
\end{tabular}

The contribution ratios of the first component (the Tsallis statistics) are in the range from 0.705 to 0.912 , which are listed only in Table 1 and not shown in plot to avoid trivialness. The main contribution ratios reflect the strong power of the Tsallis statistics in the fitting process for the $p_{T}$ spectra of heavy quarkonia in various rapidity regions. Meanwhile, the contribution ratios $(1-k)$ of the second component (the Hagedorn function) are considerable. The contribution ratios do not show particular behaviors, but almost invariant with rapidity. This means that the impact between the two "participant" quarks is very violent. The effects of other factors such as the rapidity region are not dominant.

We would like to point out that the Hagedorn function is indeed needed, though the Tsallis statistics has power-law tail in high $p_{T}$ region. In fact, if we use the Tsallis statistics to fit the spectra in high $p_{T}$ region, the fit in low $p_{T}$ region will be failed. Contrarily, if the low $p_{T}$ region is fixed, the high $p_{T}$ region will be on the wrong way. In our opinion, the Tsallis statistics should fit the spectra from 0 to the range as widely as possible. The Hagedorn function should fit the spectra in high $p_{T}$ region, though its contribution is from 0 to high $p_{T}$ region.
Comparing with that in $p p$ collisions, the parameters from the spectra in $p-\mathrm{Pb}$ collisions do not show particular behaviors. This means that the cold nuclear effect affects mainly the normalizations of $p_{T}$ spectra of heavy quarkonia, but not the shapes. In fact, the heavy quarkonia are produced in the process of violent impact between two "participant" quarks in the considered collisions. Not only the spectator nucleons but also the "spectator" quarks do not affect largely the shapes of $p_{T}$ spectra of heavy quarkonia. Naturally, the cold nuclear effect in lead-lead and other nucleus-nucleus collisions has no large effect on the shapes of $p_{T}$ spectra of heavy quarkonia.

It should be noted that a given free parameter for prompt $J / \psi$ and $J / \psi$ from $b$ leads to similar result or small difference at one energy, and large difference at another energy. If small difference is explained by statistical fluctuation in the data, large difference can be explained by dynamical reason. For example, the results for prompt $J / \psi$ and $J / \psi$ from $b$ at 7 and $8 \mathrm{TeV}$ lead to similar $p_{0}$ and $n$, and the results at $13 \mathrm{TeV}$ lead to different $n$ in both cases. It is possible that the dynamical mechanism at $13 \mathrm{TeV}$ is different from that at 7 and $8 \mathrm{TeV}$ due to different energies. The large difference should be studied in the near future by the more accurate pQCD method. 


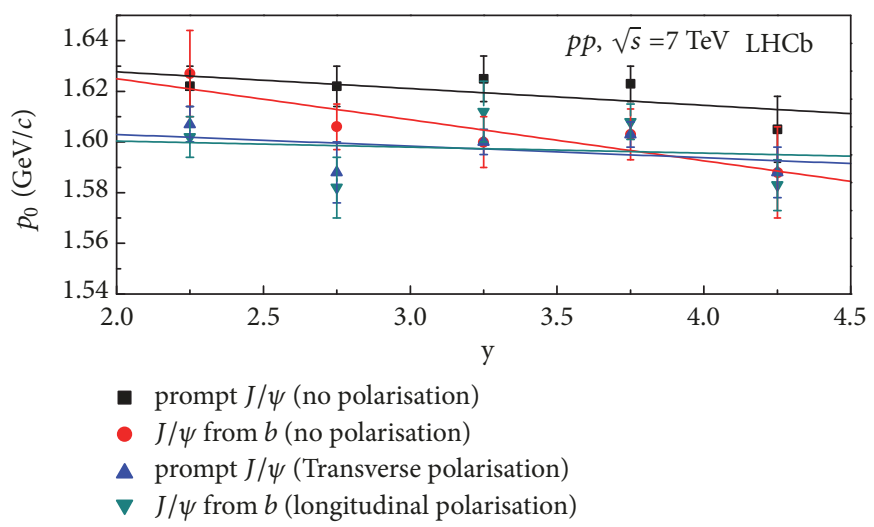

(a)

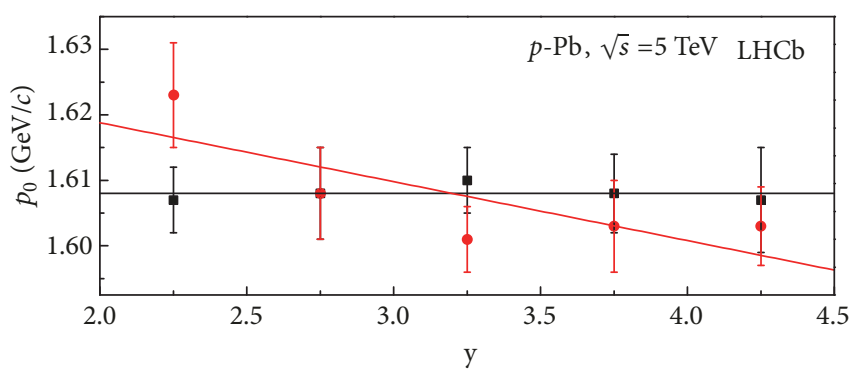

- prompt $J / \psi$

- $J / \psi$ from $b$

(b)

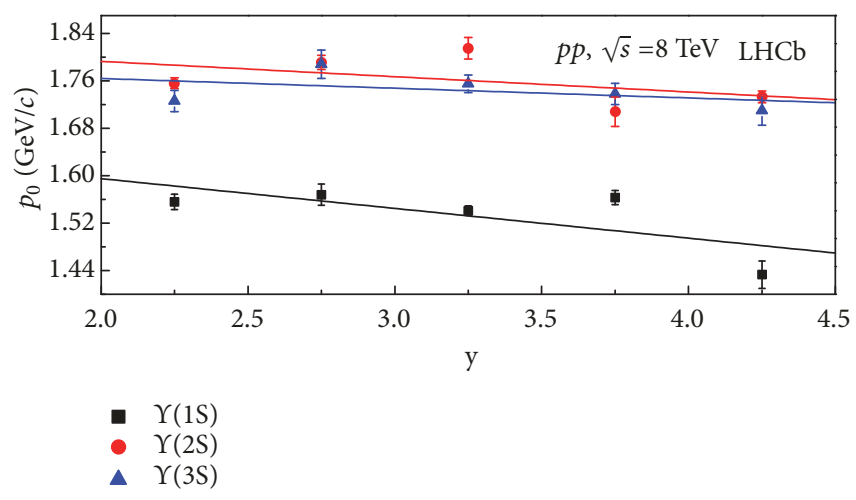

(d)

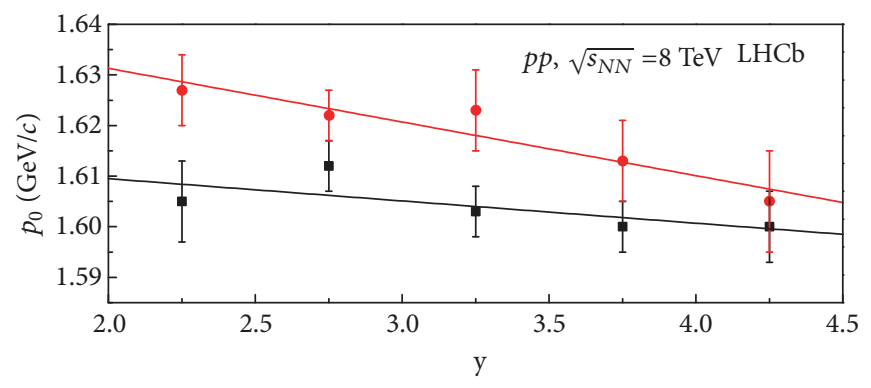

- prompt $J / \psi$

$J / \psi$ from $b$

(c)

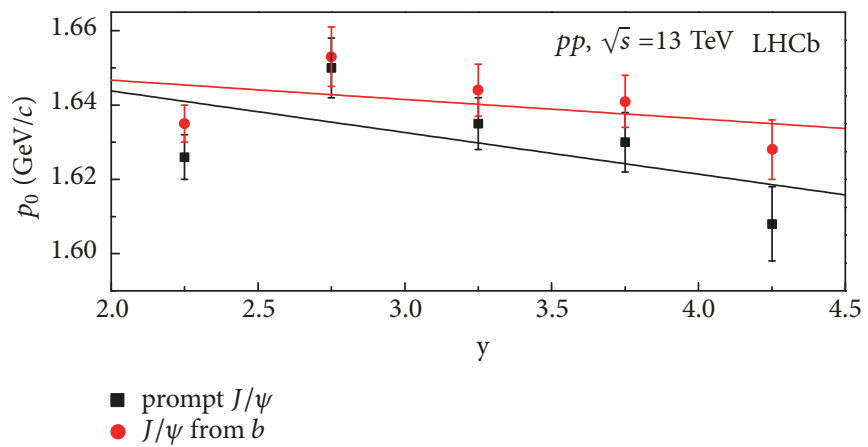

(e)

Figure 8: The same as Figure 6, but showing the relationship between $p_{0}$ and $y$.

In addition, in which concerns prompt $J / \psi$, most of free parameters show no or very small dependence on rapidity, except maybe at $13 \mathrm{TeV}$. This seems that the difference, if existent, shown by the data is contained either in the normalization or the previously mentioned subtracted midvalue of the given rapidity range. In fact, both the factors do not affect the free parameters which are only determined by the shapes of $p_{T}$ spectra. The treatment shifted the given rapidity range to the mid-rapidity region is necessary due to the fact that the kinetic energy of directional movement should not be included in the temperature.
To analyze further the behaviors of parameters, Figures 10 and 11 present the dependence of mean $p_{T}\left(\left\langle p_{T}\right\rangle\right)$ and ratio of root-mean-square $p_{T}\left(\sqrt{\left\langle p_{T}^{2}\right\rangle}\right)$ to $\sqrt{2}$ on rapidity, respectively. The symbols represent the values of $\left\langle p_{T}\right\rangle$ and $\sqrt{\left\langle p_{T}^{2}\right\rangle / 2}$ obtained from the curves in Figures 1-5. The lines are the fitted results of linear functions, though some of them do not obey the linear relationship. The intercepts, slopes, and $\chi^{2} /$ dof corresponding to these lines are listed in Table 2. One can see the decreasing trends of the considered quantities with the increase of rapidity. These trends also 


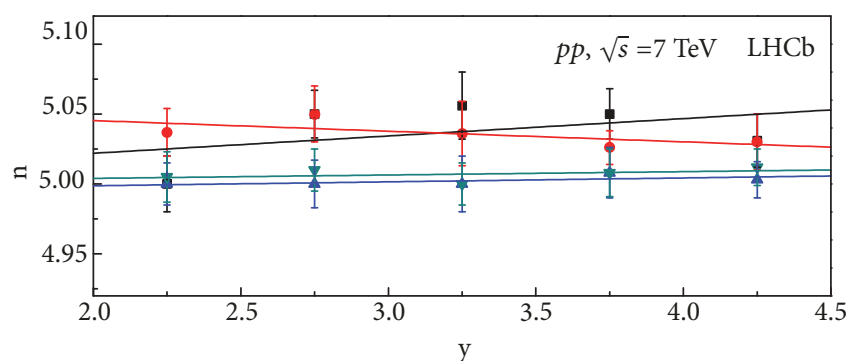

- prompt $J / \psi$ (no polarisation)

- $J / \psi$ from $b$ (no polarisation)

$\Delta$ prompt $J / \psi$ (Transverse polarisation)

$\boldsymbol{\nabla} J / \psi$ from $b$ (longitudinal polarisation)

(a)

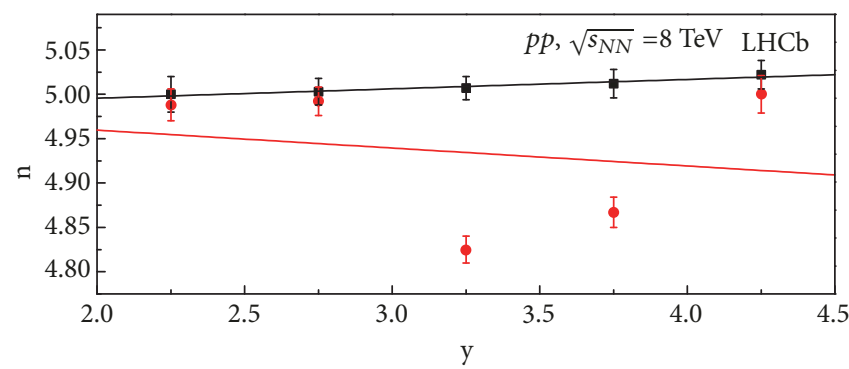

prompt $J / \psi$

- $J / \psi$ from $b$

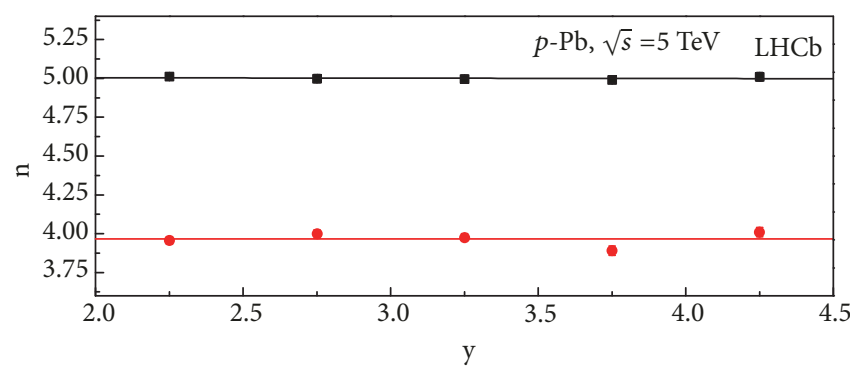

arompt $J / \psi$

- $J / \psi$ from $b$

(b)

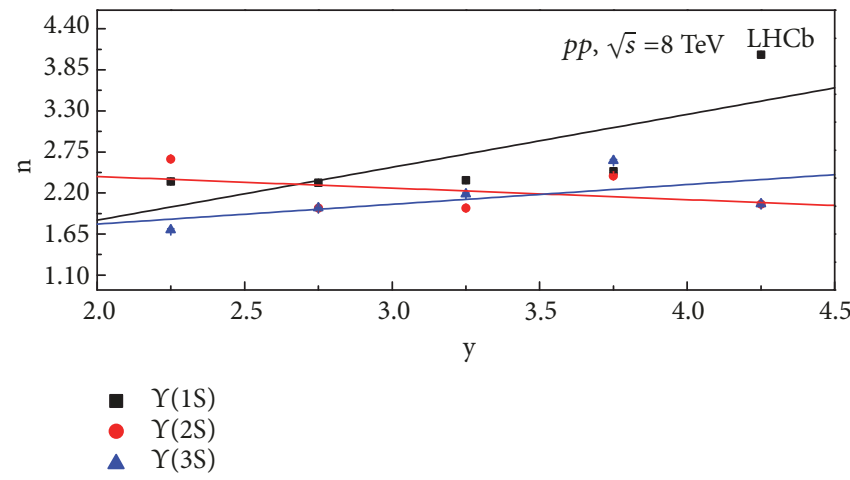

(d)

(c)

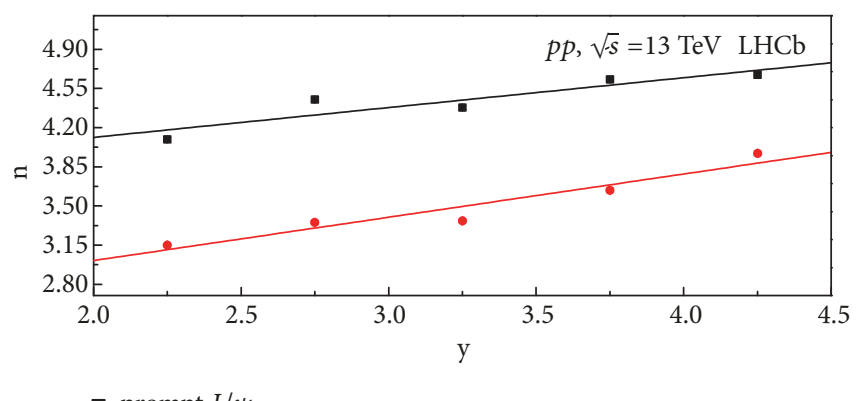

- prompt $J / \psi$

- $J / \psi$ from $b$

(e)

FIGURE 9: The same as Figure 6, but showing the relationship between $n$ and $y$.

render decreasing excitation degree of the interacting system with the increase of rapidity.

According to [37-39], if the initial temperature $\left(T_{i}\right)$ of the interacting system is approximately described by $\sqrt{\left\langle p_{T}^{2}\right\rangle / 2}$, Figure 11 shows that $T_{i}$ decreases when the rapidity increases due to less energy deposition in very forward rapidity region. At the same or similar LHC energy, $T_{i}$ extracted from the spectra of $J / \psi$ (or $\Upsilon$ ) mesons is about 6 (or 12) times of that $(\sim 0.4 \mathrm{GeV})$ extracted from the spectra of pion mesons [40]. As the quantities which are independent of models, both $\left\langle p_{T}\right\rangle$ and $T_{i}$ are very important in the understanding the excitation degree of interacting system. More investigations on $\left\langle p_{T}\right\rangle$ and $T_{i}$ are needed due to their importance.
Similar to most of free parameters, the derived quantities $\left\langle p_{T}\right\rangle$ and $T_{i}$ from the model also show no or very small dependence on rapidity. Although $\left\langle p_{T}\right\rangle$ and $T_{i}$ are calculated from the model, they depend mainly on the data. In fact, $\left\langle p_{T}\right\rangle$ and $T_{i}$ are independent of models. The similar dependence on rapidity for most of free parameters and derived quantities renders that the treatment previously mentioned subtracted mid-value of the given rapidity range is correct.

Before conclusions, we would like to point out that the present work is a new analysis on the LHCb data [15-18] analyzed in our recent work [41] which does not fit very well the spectra in high $p_{T}$ region. In the present work, to fit the spectra in high $p_{T}$ region, the inverse power-law [32-34] (Hagedorn function [35]) is used as the second component 


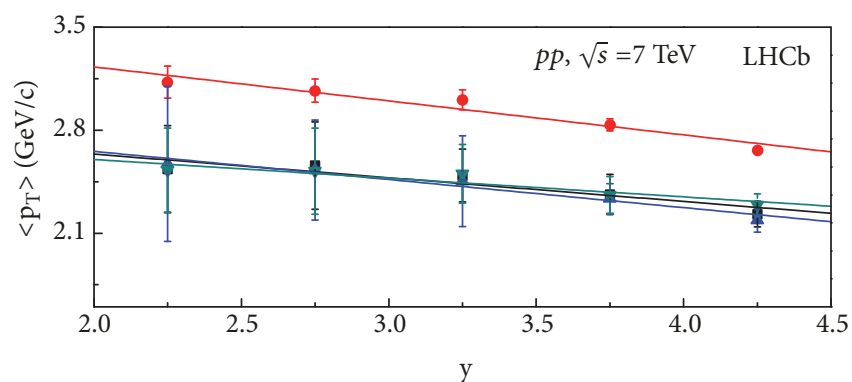

- prompt $J / \psi$ (no polarisation)

- $J / \psi$ from $b$ (no polarisation)

$\Delta$ prompt $J / \psi$ (Transverse polarisation)

$\nabla J / \psi$ from $b$ (longitudinal polarisation)

(a)

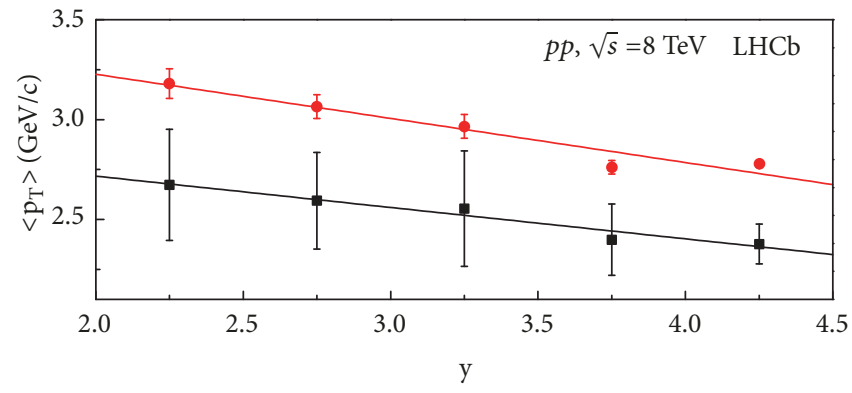

- prompt $J / \psi$

- $J / \psi$ from $b$

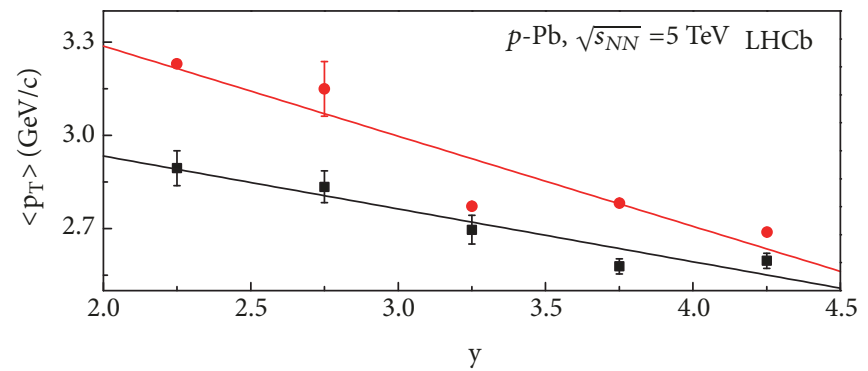

prompt $J / \psi$

- $J / \psi$ from $b$

(b)

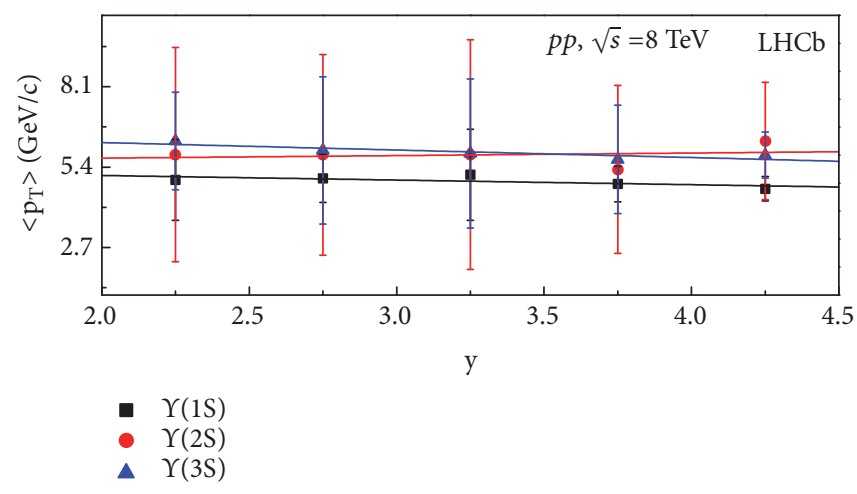

(d)

(c)

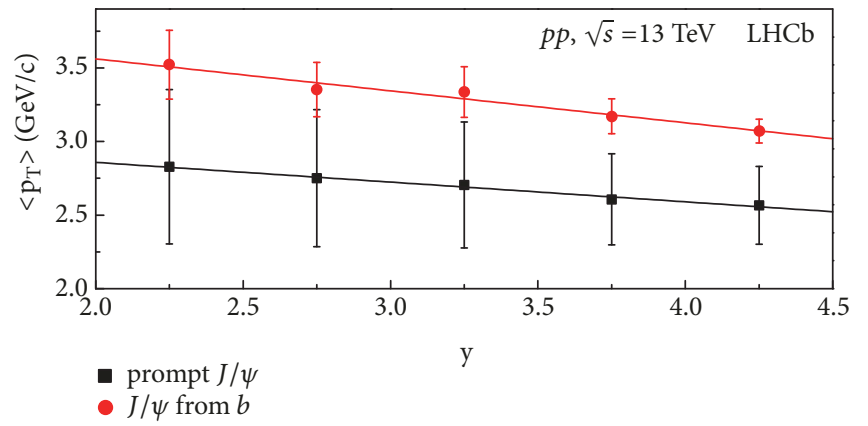

(e)

FIGURE 10: The same as Figure 6, but showing the relationship between $\left\langle p_{T}\right\rangle$ and $y$.

to structure a superposition with the Tsallis statistics [2731]. Intuitively, the fitted results of the two-component model are obviously improved, though three more parameters are introduced. Anyhow, the behaviors of more parameters are revealed in the present work.

\section{Conclusions}

We summarize here our main observations and conclusions.

The rapidity dependent transverse momentum spectra of heavy quarkonia $(J / \psi$ and $\Upsilon$ mesons) produced in small collision systems ( $p p$ and $p$-Pb collisions) at high energy $(\sqrt{s}$ $\left.\left(\sqrt{s_{\mathrm{NN}}}\right)=5-13 \mathrm{TeV}\right)$ have been analyzed by a two-component statistical model which is based on the Tsallis statistics and inverse power-law. The experimental data measured by the LHCb Collaboration at the LHC are well fitted by the model results. The related parameters are obtained and the dependence of parameters on rapidity is analyzed.

The invariant or slight decreasing temperature parameter renders that the excitation degree of the interacting system keeps invariant or slight decreasing trend with the increase of rapidity. The heavy quarkonia produce much earlier than light particles due to very high temperature from the spectra of heavy quarkonia. The considered interacting system stays approximately at the (local) equilibrium state due to the entropy index being close to 1 . The decreasing entropy index renders that the system stays at a more equilibrium state in the very forward rapidity region. 


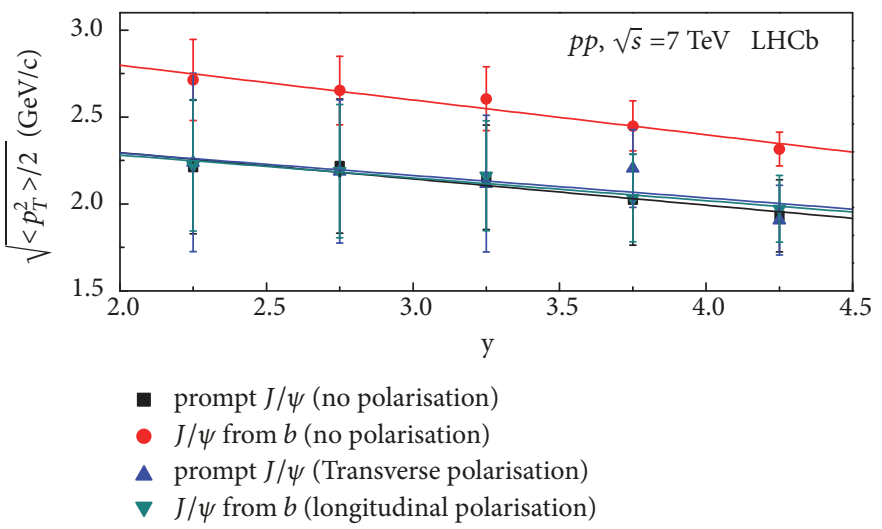

(a)

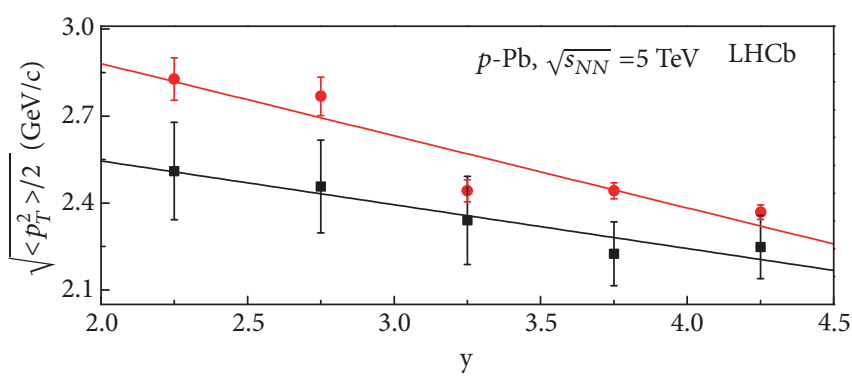

- prompt $J / \psi$

- $J / \psi$ from $b$

(b)

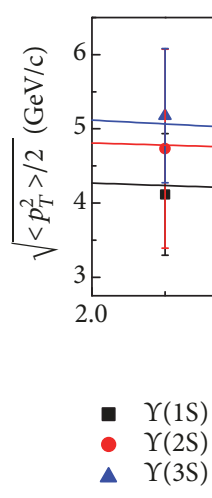

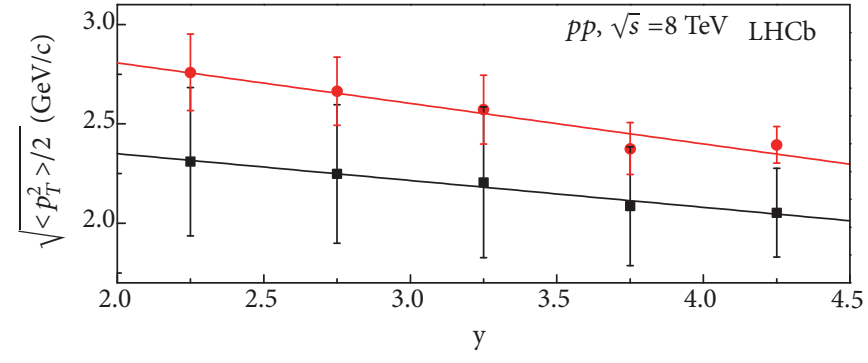

- prompt $J / \psi$

- $J / \psi$ from $b$

(c)

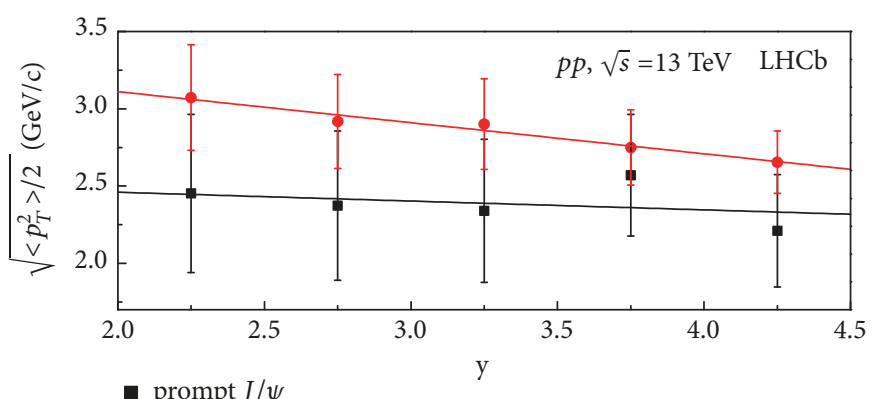

prompt $J / \psi$

- $J / \psi$ from $b$

(d)

(e)

FIGURE 11: The same as Figure 6, but showing the relationship between $\sqrt{\left\langle p_{T}^{2}\right\rangle / 2}$ and $y$.

A slightly narrow $p_{T}$ range in the very forward rapidity region is observed due to the decreasing $p_{0}$ and increasing $n$. This means that the scattering strength of the interacting system decreases slightly with the increase of rapidity. The contribution ratio of the Tsallis statistics is close to 1 . This reflects the strong power of the Tsallis statistics in the fitting process for the $p_{T}$ spectra of heavy quarkonia, in various rapidity regions. The impact between the two "participant" quarks is very violent. Other factors do not play dominant functions.

The cold nuclear effect does not affect largely the production of heavy quarkonia due to the fact that the parameters from the spectra in $p-\mathrm{Pb}$ collisions do not show particular behaviors, comparing with that in $p p$ collisions. Not only the spectator nucleons but also the "spectator" quarks do not affect largely the production of heavy quarkonia. The heavy quarkonia are only produced in the process of violent impact between two "participant" quarks in the considered collisions.

The mean transverse momentum $\left\langle p_{T}\right\rangle$, root-meansquare transverse momentum $\sqrt{\left\langle p_{T}^{2}\right\rangle}$, and initial temperature $\sqrt{\left\langle p_{T}^{2}\right\rangle / 2}$ decrease with the increase of rapidity due to the fact that less energy deposition appears in very forward rapidity region. At the same or similar LHC energy, the initial 
temperature extracted from the spectra of $J / \psi$ (or $\Upsilon$ ) mesons is about 6 (or 12) times of that $(\sim 0.4 \mathrm{GeV})$ extracted from the spectra of pion mesons.

\section{Data Availability}

The data used to support the findings of this study are included within the article.

\section{Ethical Approval}

The authors declare that they are in compliance with ethical standards regarding the content of this paper.

\section{Conflicts of Interest}

The authors declare that they have no conflicts of interest regarding the publication of this paper.

\section{Acknowledgments}

Li-Na Gao acknowledges the financial supports from the National Natural Science Foundation of China under Grant no. 11847003, the Doctoral Scientific Research Foundation of Taiyuan Normal University under Grant no. I170167, and the Doctoral Scientific Research Foundation of Shanxi Province under Grant no. I170269. Other authors acknowledge the financial supports from the National Natural Science Foundation of China under Grant nos. 11575103 and 11847311, the Shanxi Provincial Natural Science Foundation under Grant no. 201701D121005, and the Fund for Shanxi "1331 Project" Key Subjects Construction.

\section{References}

[1] B. I. Abelev, M. M. Aggarwal, Z. Ahammed et al., "Systematic measurements of identified particle spectra in $p p, d+A u$, and $\mathrm{Au}+\mathrm{Au}$ collisions at the STAR detector," Physical Review C, vol. 79, Article ID 034909, 2009.

[2] B. I. Abelev, M. Planinić, and N. Poljak, "Identified particle production, azimuthal anisotropy, and interferometry measurements in $\mathrm{Au}+\mathrm{Au}$ collisions at $\sqrt{s_{N N}}=9.2 \mathrm{GeV}$," Physical Review C, vol. 81, Article ID 024911, 2010.

[3] E. Schnedermann, J. Sollfrank, and U. Heinz, "Thermal phenomenology of hadrons from $200 \mathrm{~A} \mathrm{GeV} \mathrm{S+S} \mathrm{collisions,"} \mathrm{Physi-}$ cal Review C, vol. 48, pp. 2462-2475, 1993.

[4] G. Wilk and Z. Włodarczyk, "Interpretation of the nonextensivity parameter $q$ in some applications of Tsallis statistics and Levy distributions," Physical Review Letters, vol. 84, pp. 27702773, 2000.

[5] J. Adams et al., " $K(892)^{*}$ resonance production in $\mathrm{Au}+\mathrm{Au}$ and $p+p$ collisions at $\sqrt{s_{N N}}=200 \mathrm{GeV}$," Physical Review C, vol. 71, Article ID 064902, 2005.

[6] L.-N. Gao, F.-H. Liu, and R. A. Lacey, "Excitation functions of parameters in Erlang distribution, Schwinger mechanism, and tsallis statistics in RHIC BES program," The European Physical Journal A, vol. 52, p. 137, 2016.

[7] C. Tsallis, "Possible generalization of boltzmann-gibbs statistics," Journal of Statistical Physics, vol. 52, pp. 479-487, 1988.
[8] C. Tsallis, "Nonadditive entropy and nonextensive statistical mechanics -an overview after 20 years," Brazilian Journal of Physics, vol. 39, p. 337, 2009.

[9] W. M. Alberico, P. Czerski, A. Lavagno, M. Nardi, and V. Somá, "Signals of non-extensive statistical mechanics in high energy nuclear collisions," Physica A: Statistical Mechanics and its Applications, vol. 387, pp. 467-475, 2008.

[10] W. M. Alberico and A. Lavagno, "Non-extensive statistical effects in high-energy collisions," The European Physical Journal A, vol. 40, p. 313, 2009 .

[11] G. Wilk and Z. Wlodarezyk, "Multiplicity fluctuations due to the temperature fluctuations in high-energy nuclear collisions," Physical Review C, vol. 79, Article ID 054903, 2009.

[12] J. Cleymans and D. Worku, "Relativistic thermodynamics: transverse momentum distributions in high-energy physics," The European Physical Journal A, vol. 48, p. 160, 2012.

[13] C. Y. Wong and G. Wilk, "Tsallis fits to $p_{T}$ spectra and multiple hard scattering in $p p$ collisions at the LHC," Physical Review D, vol. 87, Article ID 114007, 2013.

[14] J. Cleymans, G. I. Lykasov, A. S. Parvan, A. S. Sorin, O. V. Teryaev, and D. Worku, "Systematic properties of the Tsallis distribution: Energy dependence of parameters in high energy $p-p$ collisions," Physics Letters B, vol. 723, p. 351, 2013.

[15] R. Aaij, B. Adeva, M. Adinolfi et al., "Measurement of $J / \Psi$ production in $p p$ collisions at $\sqrt{s}=7 \mathrm{TeV}$," The European Physical Journal C, vol. 71, p. 1645, 2011.

[16] R. Aaij, C. Abellan Beteta, B. Adeva et al., "Production of $J / \psi$ and $\Upsilon$ mesons in pp collisions at $\sqrt{s}=8 \mathrm{TeV}$," Journal of High Energy Physics, vol. 2013, no. 06, article no. 064, 2013.

[17] R. Aaij, B. Adeva, M. Adinolfi et al., "Study of $J / \psi$ production and cold nuclear matter effects in pPb collisions at $\sqrt{s_{N N}}=5$ TeV," Journal of High Energy Physics, vol. 2014, no. 02, article no. 072, 2014.

[18] R. Aaij, B. Adeva, M. Adinolfi et al., "Measurement of forward $\mathrm{J} / \psi$ production cross-sections in pp collisions at $\sqrt{s}=13 \mathrm{TeV}$," Journal of High Energy Physics, vol. 2015, no. 10, article no. 172, 2015.

[19] J. Adam, D. Adamová, M. M. Aggarwal et al., "Differential studies of inclusive $\mathrm{J} / \psi$ and $\psi(2 \mathrm{~S})$ production at forward rapidity in $\mathrm{Pb}-\mathrm{Pb}$ collisions at $\sqrt{s_{N N}}=2.76 \mathrm{TeV}$,' Journal of High Energy Physics, vol. 2016, no. 05, article no. 179, 2016.

[20] F.-H. Liu, "Particle production in $\mathrm{Au}-\mathrm{Au}$ collisions at RHIC energies," Physics Letters B, vol. 583, p. 68, 2004.

[21] F.-H. Liu, "Dependence of charged particle pseudorapidity distributions on centrality and energy in $p(d) A$ collisions at high energies," Physical Review C, vol. 78, Article ID 014902, 2008.

[22] F.-H. Liu, "Unified description of multiplicity distributions of final-state particles produced in collisions at high energies," Nuclear Physics A, vol. 810, pp. 159-172, 2008.

[23] S. Ostapchenko, H. J. Drescher, F. M. Liu, T. Pierog, K. Werner, and J. Phys, "Consistent treatment of soft and hard processes in hadronic interactions," Journal of Physics G, vol. 28, p. 2597, 2002.

[24] M. G. Ryskin, A. D. Martin, and V. A. Khoze, "High-energy strong interactions: from 'hard' to 'soft,' The European Physical Journal C, vol. 71, p. 1617, 2011.

[25] I. M. Dremin and V. A. Nechitailo, "Soft multiple parton interactions as seen in multiplicity distributions at Tevatron and LHC," Physical Review D, vol. 84, Article ID 034026, 2011. 
[26] A. A. Grinyuk, A. V. Lipatov, G. I. Lykasov, and N. P. Zotov, "Transition between soft physics at the LHC and low- $x$ physics at HERA," Physical Review D, vol. 87, Article ID 074017, 2013.

[27] J. Cleymans and D. Worku, "The Tsallis distribution in protonproton collisions at $\sqrt{s}=0.9 \mathrm{TeV}$ at the LHC," Journal of Physics $G$, vol. 39, Article ID 025006, 2012.

[28] M. D. Azmi and J. Cleymans, "Transverse momentum distributions in proton-proton collisions at LHC energies and Tsallis thermodynamics," Journal of Physics G, vol. 41, Article ID 065001, 2014.

[29] B.-C. Li, Y.-Z. Wang, and F.-H. Liu, "Formulation of transverse mass distributions in $\mathrm{Au}-\mathrm{Au}$ collisions at $\sqrt{s_{N N}}=200 \mathrm{CeV} /$ nucleon," Physics Letters B, vol. 725, p. 352, 2013.

[30] M. Rybczynski and Z. Wlodarczyk, "Tsallis statistics approach to the transverse momentum distributions in p-p collisions," The European Physical Journal C, vol. 74, p. 2785, 2014.

[31] H. Zheng and L.-L. Zhu, "Comparing the tsallis distribution with and without thermodynamical description in $p+p$ collisions," Advances in High Energy Physics, vol. 2016, Article ID 9632126, 10 pages, 2016.

[32] R. Odorico, "Does a transverse energy trigger actually trigger on large- $P_{T}$ jets?" Physics Letters B, vol. 118, p. 151, 1982.

[33] G. Arnison, A. Astbury, and B. Aubert, “Transverse momentum spectra for charged particles at the CERN proton-antiproton collider," Physics Letters B, vol. 118, p. 167, 1982.

[34] T. Mizoguchi, M. Biyajima, and N. Suzuki, "Analyses of whole transverse momentum distributions in $p \bar{p}$ and $p p$ collisions by using a modified version of Hagedorn's formula," International Journal of Modern Physics A, vol. 32, Article ID 1750057, 2017.

[35] R. Hagedorn, "Multiplicities, $p_{T}$ distributions and the expected hadron-quark-gluon phase transition," La Rivista del Nuovo Cimento, vol. 6, pp. 1-50, 1983.

[36] H.-L. Lao, F.-H. Liu, B.-C. Li, M.-Y. Duan, and R. A. Lacey, "Examining the model dependence of the determination of kinetic freeze-out temperature and transverse flow velocity in small collision system," Nuclear Science and Techniques, vol. 29, p. 164, 2018.

[37] L. J. Gutay, A. S. Hirsch, R. P. Scharenberg, B. K. Srivastava, and C. Pajares, "De-confinement in small systems: Clustering of color sources in high multiplicity $\bar{p} p$ collisions at $\sqrt{s}=1.8$ TeV," International Journal of Modern Physics E, vol. 24, Article ID 1550101, 2015.

[38] A. S. Hirsch, C. Pajares, R. P. Scharenberg, and B. K. Srivastava, "De-Confinement in high multiplicity proton-proton collisions at LHC energies," https://arxiv.org/abs/1803.02301, 2018.

[39] P. Sahoo, S. De, S. K. Tiwari, and R. Sahoo, "Energy and centrality dependent study of deconfinement phase transition in a color string percolation approach at RHIC energies," The European Physical Journal A, vol. 54, p. 136, 2018.

[40] L.-L. Li and F.-H. Liu, "Excitation functions of kinetic freezeout temperature and transverse flow velocity in proton-proton collisions," https://arxiv.org/abs/1805.03342, 2018.

[41] B.-C. Li, T. Bai, Y.-Y. Guo et al., "On $J / \psi$ and $\Upsilon$ transverse momentum distributions in high energy collisions," Advances in High Energy Physics, vol. 2017, Article ID 9383540, 7 pages, 2017. 

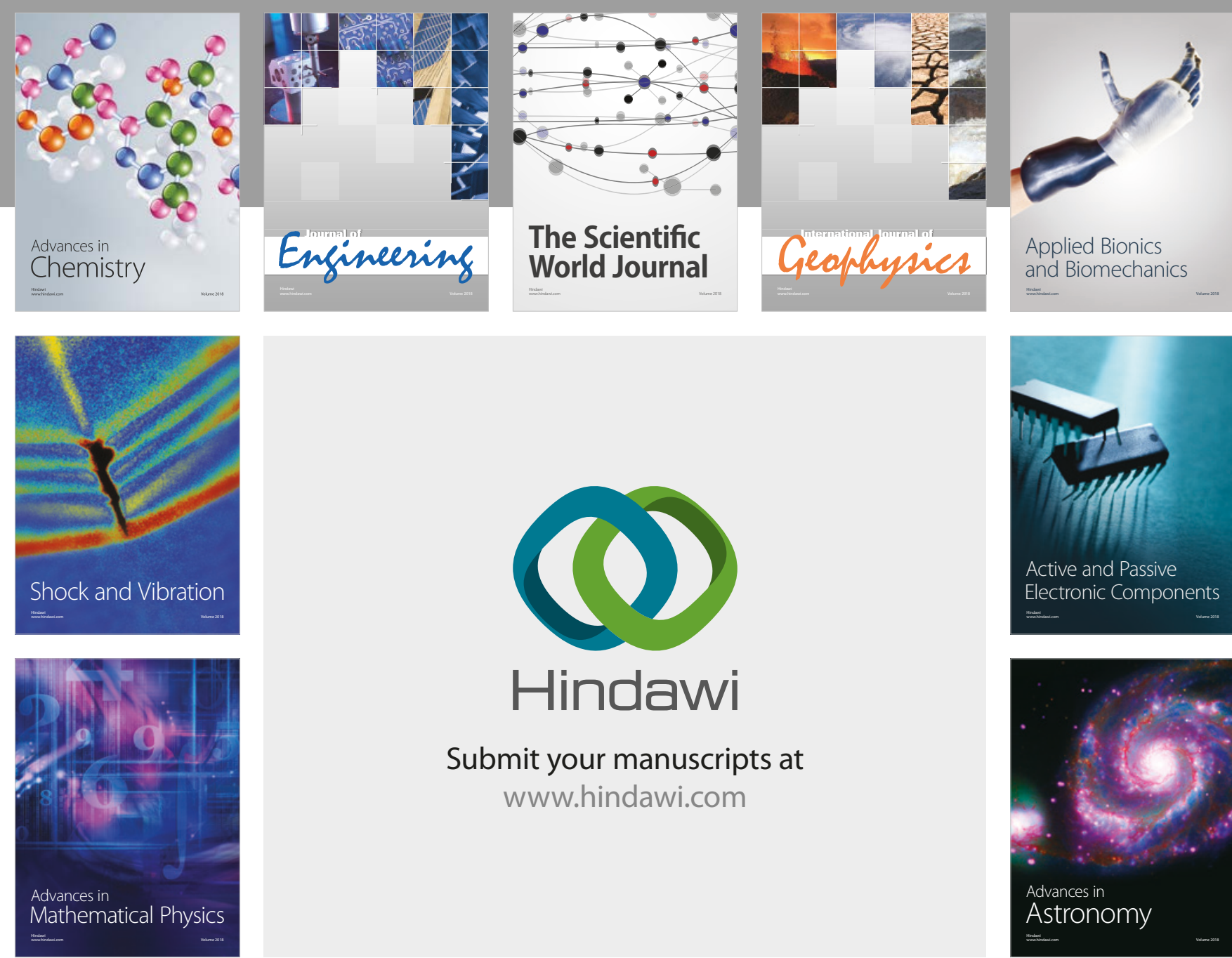

Submit your manuscripts at

www.hindawi.com

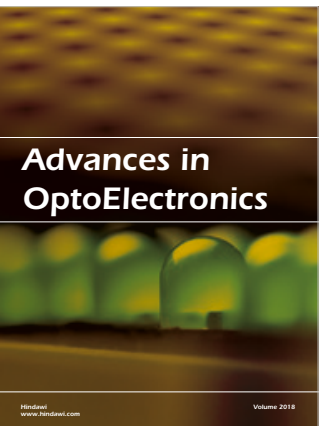

\section{Rotcting Machinery}
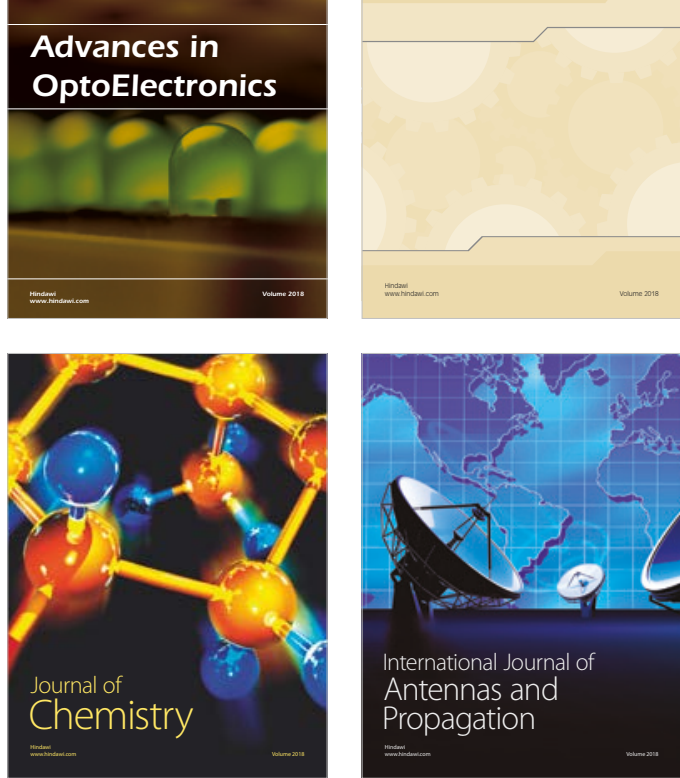

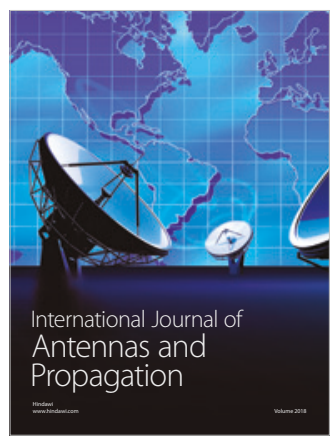

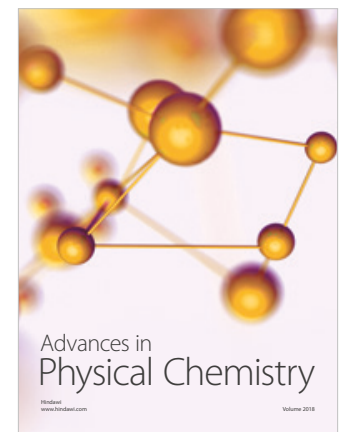

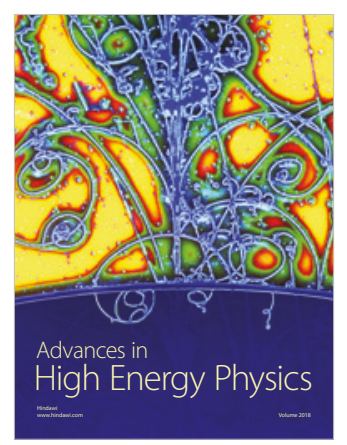

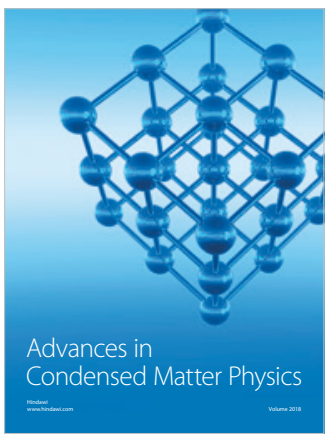

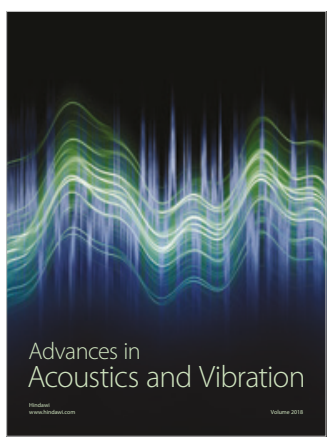

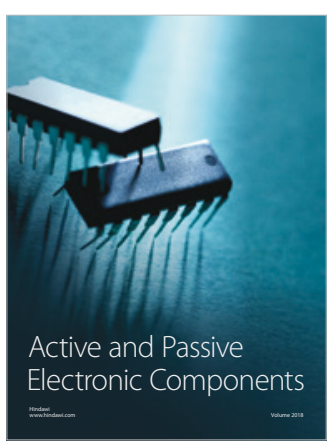
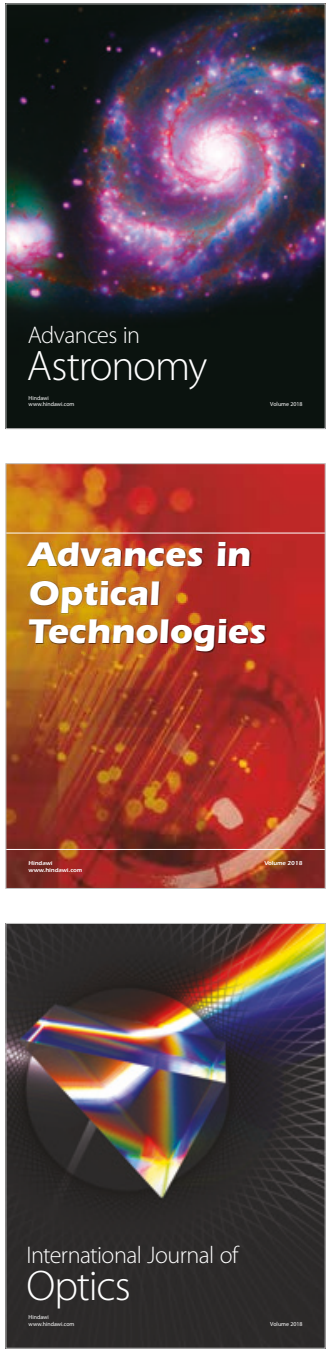